\title{
Affinage de l'emmental : dynamique des populations bactériennes et évolution de la composition de la phase aqueuse
}

\author{
Anne Thierry*, Delphine Salvat-Brunaud, Marie-Noëlle Madec, \\ Françoise Michel, Jean-Louis Maubois
}

Laboratoire de recherches de technologie laitière, Inra, 65, rue de Saint-Brieuc, 35042 Rennes cedex, France

(Reçu le 20 octobre 1997 ; accepté le 17 mars 1998)

\begin{abstract}
Swiss cheese ripening: dynamics of bacterial populations and evolution of the aqueous phase composition for three industrial cheeses. To understand and control cheese ripening, it is essential to know the kinetics of the microbial and biochemical changes occurring during ripening. For three Swiss cheeses industrially manufactured, differing particularly in ripening times and temperatures, we determined throughout the ripening the dynamics of starter and non-starter flora and the changes in the composition of cheese aqueous phase extracted by pressing. Three flora succeeded one another during ripening: thermophilic lactic starters, non-starter lactic acid bacteria and propionibacteria. The viability of lactic starters fell during ripening from $10^{9}$ to $10^{8}-10^{5} \mathrm{cfu} \cdot \mathrm{g}^{-1}$ more or less rapidly, depending on the cheeses. Propionibacteria reached $10^{8}$ to $410^{8} \mathrm{cfu} \cdot \mathrm{g}^{-1}$ at the end of ripening. Non-starter lactic acid bacteria grew from the beginning of ripening and reached populations as high as $10^{8}$ to $510^{8} \mathrm{cfu} \cdot \mathrm{g}^{-1}$. They were composed of heterofermentative lactobacilli, Lactobacillus paracasei constituting the predominant species (56\% to $88 \%$ of the isolates). The aqueous phase composition showed similar changes for the three cheeses. Nitrogen matter increased from 13-18 to $54-68 \mathrm{~g} \cdot \mathrm{kg}^{-1}$ during ripening, with a rate depending on the ripening room temperature. Free amino acids increased from $10-29 \%$ to $45-47 \%$ of the nitrogen matter of the aqueous phase, but their relative proportion remained essentially constant, apart from amino acids catabolised by bacteria (Arg and Asp in particular). Most mineral exchanges between curd and aqueous phase occurred before the ripening during the curd acidification, resulting in high levels of $\mathrm{Ca}, \mathrm{Mg}$ and $\mathrm{P}$ in aqueous phase (respectively about $6.5,0.65$ and $1.2 \mathrm{~g} \cdot \mathrm{kg}^{-1}$ ). Mineral composition showed only minor changes during ripening. Citrate (1.7-2.4 $\mathrm{g} \cdot \mathrm{kg}^{-1}$ in aqueous phase at the beginning of ripening) was consumed during cold room ripening, presumably by non-starter flora. Lactate $\left(34-36 \mathrm{~g} \cdot \mathrm{kg}^{-1}\right)$ decreased only by 1 to $2 \mathrm{~g} \cdot \mathrm{kg}^{-1}$ in the three cheeses. (c) Inra/Elsevier, Paris.
\end{abstract}

Swiss cheese / ripening / bacteria / aqueous phase / composition

\footnotetext{
* Correspondance et tirés à part. E-mail: athierry@ labtechno.roazhon.inra.fr
} 
Résumé - Pour comprendre et maîtriser l'affinage d'un fromage, il est nécessaire de connaître la cinétique des principales transformations microbiennes et biochimiques. Pour trois séries d'emmental de fabrication industrielle, qui différaient notamment par les températures et durées en cave d'affinage, nous avons suivi au cours de l'affinage l'évolution des flores levains et non levains et, parallèlement, celle de la composition de la phase aqueuse extraite par pressage. Trois flores se succédaient au cours de l'affinage : les levains lactiques thermophiles, des bactéries lactiques non levains puis les bactéries propioniques. La viabilité des levains lactiques thermophiles chutait au cours de l'affinage, avec une ampleur et une cinétique différant d'une série d'emmental à l'autre. La flore propionique atteignait $10^{8}$ à $410^{8} \mathrm{ufc} \cdot \mathrm{g}^{-1} \mathrm{en}$ fin d'affinage. De plus, dès le début de l'affinage, une flore lactique non levain se développait, composée de lactobacilles hétérofermentaires principalement représentés par Lactobacillus paracasei. La composition de la phase aqueuse évoluait de façon voisine pour les 3 séries d'emmental. La matière azotée de la phase aqueuse passait de 13-18 à $54-68 \mathrm{~g} \mathrm{~kg}^{-1}$ au cours de l'affinage, avec des cinétiques étroitement liées aux températures des caves d'affinage. La proportion d'acides aminés libres passait de 10-29 à 45-47\%, mais le profil en acides aminés libres variait relativement peu au cours de l'affinage, à l'exception des acides aminés dégradés par catabolisme bactérien (Arg et Asp en particulier). La composition minérale ne présentait que de faibles variations au cours de l'affinage. L'essentiel des échanges minéraux entre le réseau paracaséinique et la phase aqueuse du fromage avait lieu avant l'affinage lors de l'acidification sous presse, conduisant à des teneurs élevées en $\mathrm{Ca}, \mathrm{Mg}$ et $\mathrm{P}$ dans la phase aqueuse (respectivement environ 6,5, 0,65 et $\left.1,2 \mathrm{~g} \cdot \mathrm{kg}^{-1}\right)$. Le citrate $\left(1,7 \mathrm{à} 2,4 \mathrm{~g} \cdot \mathrm{kg}^{-1}\right.$ dans la phase aqueuse en début d'affinage) était consommé en cave tempérée, vraisemblablement par la flore non levain. Le lactate (34-36 $\mathrm{g}^{\mathrm{kg}}{ }^{-1} \mathrm{de}$ phase aqueuse), fermenté par les bactéries propioniques en cave chaude, ne diminuait que de 1 à $2 \mathrm{~g} \cdot \mathrm{kg}^{-1}$ dans ces 3 séries d'emmental. (C) Inra/Elsevier, Paris.

emmental / affinage / bactérie / phase aqueuse / composition

\section{INTRODUCTION}

L'emmental est le premier fromage français, avec plus de 231000 tonnes produites en 1996 [45]. Les deux tiers de sa production sont concentrés dans des unités d'une capacité annuelle supérieure à 3000 tonnes. Dix fromageries de l'Ouest de la France ont ainsi fourni $57 \%$ de la production nationale en 1996 [45]. Cette concentration va de pair avec des cadences de production élevées et exige de mieux connaître et maîtriser l'affinage, processus complexe qui, du fait des nombreuses interactions entre acteurs d'affinage, nécessite d'avoir une vision globale des changements biochimiques et de leurs liens avec les flores en présence.

Les données de la littérature sur l'emmental ne concernent cependant le plus souvent qu'un aspect de l'affinage, les plus décrits étant la fermentation propionique et la protéolyse. La fermentation propionique résulte du développement en cave chaude des bactéries propioniques, qui transforment le lac- tate produit par les bactéries lactiques thermophiles en propionate, acétate et $\mathrm{CO}_{2}$, composés indispensables à la flaveur et à l'ouverture caractéristiques de ce fromage $[32,47]$. La protéolyse dans le cas de l'emmental, repose essentiellement sur l'action combinée de la plasmine et des enzymes bactériennes, en particulier celle des lactobacilles thermophiles considérés comme les principaux acteurs microbiens de la protéolyse de l'emmental $[16,21,47]$. La cinétique de protéolyse de l'emmental reste mal connue et n'a fait l'objet que d'études ponctuelles [27, 41, 47] en particulier en ce qui concerne la libération des acides aminés libres [7, 31]. Aucune approche cinétique globale n'a, à notre connaissance, été conduite sur l'affinage de l'emmental.

La plupart des données portent en outre sur des emmentals fabriqués selon une technologie traditionnelle et affinés selon des cycles temps-température qui diffèrent fortement de ceux mis en œuvre dans les unités 
de production industrielles de l'Ouest de la France. Ces différentes technologies de fabrication et d'affinage conduisent à des fromages de composition différente en fin d'affinage. Ainsi, la fermentation propionique et la protéolyse sont beaucoup moins prononcées pour l'emmental français, affiné 7 à 13 semaines, que pour l'emmental suisse de 20 semaines $[5,47]$. Au sein même de la production nationale d'emmental, des différences de composition physico-chimique entre emmental Est-Central « Grand Cru », emmental Franc-Comtois et d'autres emmentals français ont été montrées [5].

Concernant l'évolution des flores au cours de l'affinage, les seules données disponibles concernent des fromages expérimentaux de type Comté, fabriqués à partir de lait cru $[13,50]$. Dans les unités industrielles de production, les bactéries lactiques thermophiles et les bactéries propioniques font l'objet d'un ensemencement systématique et se développent respectivement après moulage et lors de l'affinage en cave chaude. La flore non levain n'a pas été décrite pour l'emmental mais le développement d'une flore lactique non levain est vraisemblable dans la mesure où cette flore, généralement composée de lactobacilles hétérofermentaires pouvant provenir du lait comme de l'unité de fabrication, a été retrouvée en fin d'affinage dans de nombreux fromages [44]. Son impact sur l'affinage reste très mal connu mais il a été montré que l'addition de certains lactobacilles au lait de fabrication d'emmental entraîne un ralentissement de la fermentation propionique, vraisemblablement lié à la fermentation du citrate [29, $38,46]$.

L'objet de cette étude était de décrire les cinétiques des évolutions microbiologiques et biochimiques au cours de l'affinage d'emmental de fabrication industrielle. Nous avons choisi de caractériser les principaux changements biochimiques des fromages en extrayant leur phase aqueuse par pressage, méthode dont la faisabilité avait été démontrée dans une précédente étude [42].
Ainsi, pour trois séries d'emmental fabriquées à partir de lait thermisé dans de grandes unités de production de l'Ouest de la France, nous avons quantifié l'évolution de la composition de la phase aqueuse au cours de l'affinage et, parallèlement, établi l'évolution des populations bactériennes levains et non levains.

\section{MATÉRIEL ET MÉTHODES}

\subsection{Origine des fromages}

Les fromages étudiés étaient issus de trois fabrications industrielles de l'Ouest de la France, réalisées en hiver (novembre, janvier et février) et référencées séries $\mathrm{A}, \mathrm{B}$ et $\mathrm{C}$ par la suite. Les séries $\mathrm{A}$ et $\mathrm{B}$ provenaient de la même entreprise, à 1 an d'intervalle. La fabrication et l'affinage étaient effectués selon le protocole habituel de chaque entreprise, à partir de lait thermisé ensemencé en levains lactiques thermophiles (streptocoques et lactobacilles) et en levains propioniques. Ils présentaient des différences quant aux couples temps-température mis en œuvre (tableau I). Les fromages d'une série donnée (16 meules en général), issus de la même cuve de fabrication, évoluent de façon similaire durant l'affinage [4].

\subsection{Analyses microbiologiques}

À chaque stade d'affinage étudié ( 6 à 9 selon les fabrications), un prélèvement a été effectué à mi-hauteur et à mi-rayon ( 18 à $20 \mathrm{~cm}$ du talon). Un échantillon de $10 \mathrm{~g}$ de fromage a été broyé au waring blender (Labo-Standa, Caen, France) dans $90 \mathrm{~mL}$ de citrate de sodium $20 \mathrm{~g} \cdot \mathrm{L}^{-1}$ [20] $\mathrm{La}$ répétabilité a été déterminée pour 44 dénombrements effectués à partir d'échantillons prélevés dans deux secteurs opposés de la meule et calculée selon la FIL [19]. Les lactobacilles thermophiles ont été dénombrés sur milieu MRS, $\mathrm{pH}$ abaissé à 5,4 par de l'acide acétique [18] incubé $48 \mathrm{~h}$ à $43^{\circ} \mathrm{C}$ en anaérobiose (Anaerocult A, Merck, Nogent-sur-Marne, France). Les streptocoques thermophiles ont été dénombrés sur M17 (Biokar, Beauvais, France) incubé 48 h à $43^{\circ} \mathrm{C}$ en anaérobiose ou en aérobiose. Ces deux milieux étant peu sélectifs, quelques colonies ont été systématiquement prélevées afin de contrôler la morphologie bactérienne. Les bac- 
Tableau I. Paramètres d'affinage des trois séries d'emmental étudiées.

Table I. Ripening parameters of the three Emmental cheese fabrications.

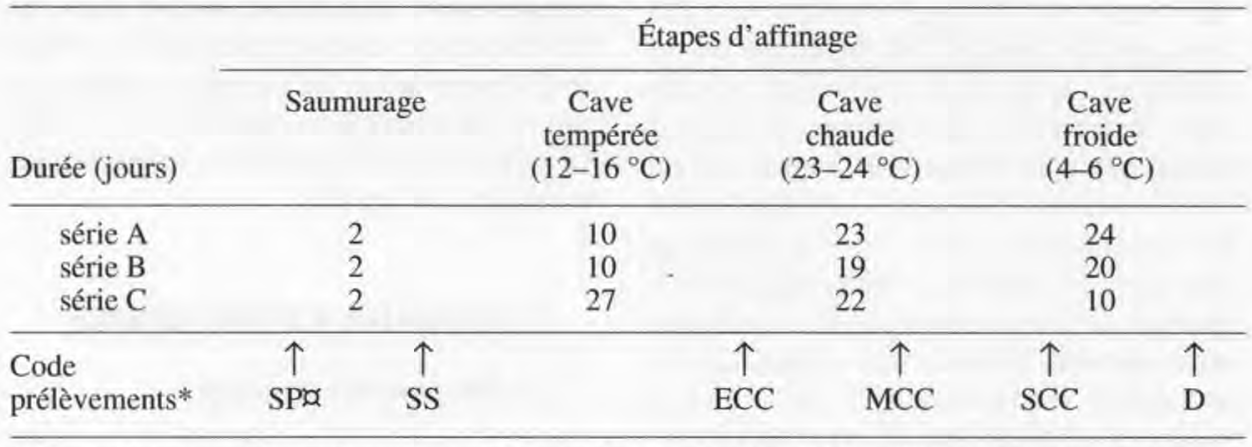

* SP : sortie presse ; SS : sortie saumure ; ECC : entrée cave chaude ; MCC : milieu cave chaude ; SCC : sortie cave chaude ; D : découpe. a Excepté pour la série C.

* Ripening stages: after the press (SP), after the brining (SS), before the warm room (ECC), mid-warm room (MCC), after the warm room (SCC) and at commercialization (D). a Except for fabrication C.

téries propioniques ont été dénombrées sur les milieux LGA et Pal Propiobac [48, 49] incubés 6 jours à $30^{\circ} \mathrm{C}$ en anaérobiose. Les lactobacilles hétérofermentaires ont été dénombrés après incubation $72 \mathrm{~h}$ à $37^{\circ} \mathrm{C}$ en anaérobiose sur le milieu FH [26], sur lequel les colonies typiques comme atypiques ont été dénombrées. Les entérocoques ont été dénombrés sur le milieu KAA incubé $24-48 \mathrm{~h}$ à $37^{\circ} \mathrm{C}$ en aérobiose [2]. Nous avons également cherché à dénombrer la flore lactique non levain totale, y compris les lactobacilles strictement hétérofermentaires qui, selon Isolini et al. [26] ne se développent pas tous sur le milieu $\mathrm{FH}$, en incubant du milieu MRS à $15^{\circ} \mathrm{C}$ pendant 10 à 14 jours en anaérobiose (milieu référencé MRS $-15^{\circ} \mathrm{C}$ par la suite). Une centaine d'isolats de la flore non levain ont été prélevés sur les milieux FH et MRS $-15^{\circ} \mathrm{C}$ pour les séries B et $\mathrm{C}$, à 3 stades d'affinage correspondant à des niveaux de flore non levain d'environ $3-4,6-7$ et $8 \log \left(\mathrm{ufc}_{\mathrm{f}} \mathrm{g}^{-1}\right)$. Après une étape de purification sur gélose MRS, ces isolats ont été caractérisés (morphologie, Gram, catalase, production de gaz à partir de glucose, croissance sur bouillon MRS à $15^{\circ} \mathrm{C}$ et $45^{\circ} \mathrm{C}$ ) et identifiés d'après leur profil fermentaire sur galeries API 50CH (Biomérieux, Marcy-l'Étoile, France).

\subsection{Extraction par pressage}

À chaque stade d'affinage étudié (tableau I), une meule de chaque série était prélevée. Trois secteurs de 2 à $2,5 \mathrm{~kg}$ constituant des échantillons représentatifs étaient pressés, soit à réception (1 secteur à chaque stade d'affinage pour les séries $\mathrm{A}$ et $\mathrm{C}$ ), soit après stockage sous vide à $-20^{\circ} \mathrm{C}$ puis décongélation 15 à $20 \mathrm{~h}$ à $4{ }^{\circ} \mathrm{C}$. Pour le pressage, le fromage était écroûté, rapé, mélangé à du sable $(1800 \mathrm{~g}$ de fromage râpé pour $3600 \mathrm{~g}$ de sable) et pressé à l'aide d'une presse hydraulique électrique, selon un protocole précédemment décrit [42]. Les phases aqueuses étaient filtrées sur papier Whatman $\mathrm{n}^{\circ}$ 541 et stockées à $-20^{\circ} \mathrm{C}$ avant analyse. À titre comparatif, deux échantillons de fromages à affinage de durée supérieure (emmental Est-Central " Grand-Cru », appelé Grand Cru par la suite, et gruyère de Comté) ont été pressés au stade découpe selon le même protocole.

\subsection{Analyses physico-chimiques}

Les analyses ont été réalisées comme décrit précédemment [42] : extrait sec par dessiccation (102-105 ${ }^{\circ} \mathrm{C}, 7$ heures), phosphore selon la FIL [17], chlorures par titrimétrie, autres minéraux (Ca, $\mathrm{Mg}, \mathrm{K}$ et $\mathrm{Na}$ ) par spectrométrie d'absorption atomique, sucres et acides organiques par HPLC, azote total (NT), azote non caséinique (NCN) et azote non protéique (NPN) par méthode de Kjeldahl et exprimés en matière azotée $(\mathrm{N} \times 6,38)$, acides aminés libres ou totaux par analyseur d'acides aminés, matière grasse des fromages par méthode acido-butyrométrique. De plus, le 
phosphore inorganique $\left(\mathrm{P}_{\mathrm{i}}\right)$ a été dosé par chromatographie ionique ainsi que, pour vérification, le citrate et les chlorures [23].

Pour les fromages, les analyses étaient effectuées sur des échantillons prélevés à mi-hauteur et à mi-rayon dans la meule. Les dosages de $\mathrm{Ca}$, $\mathrm{Mg}, \mathrm{K}$, et $\mathrm{Na}$ étaient réalisés à partir des cendres (incinération à $550^{\circ} \mathrm{C}$ pendant $3 \mathrm{~h} 30$ ) mises en solution dans $\mathrm{HCl} 1 \mathrm{~mol} \cdot \mathrm{L}^{-1}$. L'extraction des fractions azotées solubles du fromage (azote soluble à pH 4,4 (NS), NPN et acides aminés libres) était effectuée à partir d'échantillons représentatifs d'un secteur de meule [24]. Les acides acétique et propionique du fromage ont été dosés par l'ITG (Rioz, France) par chromatographie en phase gazeuse après extraction en milieu acide par un mélange d'éthers, selon Berdagué et al. [3].

\subsection{Comparaison des analyses du fromage et de sa phase aqueuse}

La quantité de phase aqueuse dans le fromage a été estimée en considérant que toute l'eau du fromage (évaluée par détermination de l'extrait $s e c)$ était solvante, c'est-à-dire contenue dans sa phase aqueuse, d'où :

$$
\begin{gathered}
\mathrm{F}_{\mathrm{pa}}(\%)=100 \times\left(1000-\mathrm{ES}_{\mathrm{f}}\right) / \\
\left(1000-\mathrm{ES}_{\mathrm{pa}}\right),
\end{gathered}
$$

où $\mathrm{F}_{\mathrm{pa}}$ est la fraction de phase aqueuse dans le fromage (\%) et $\mathrm{ES}_{\mathrm{pa}}$ et $\mathrm{ES}_{\mathrm{f}}$ sont les extraits secs respectifs de la phase aqueuse et du fromage $\left(\mathrm{g} \cdot \mathrm{kg}^{-1}\right)$.

Pour chacun des composés du fromage, on peut ainsi calculer la fraction $F_{x}$ qui se trouverait, selon la même hypothèse, dans la phase aqueuse :

$$
\mathrm{F}_{\mathrm{x}}(\%)=\mathrm{F}_{\mathrm{pa}} \times\left(\mathrm{X}_{\mathrm{pa}} / \mathrm{X}_{\mathrm{f}}\right)
$$

où $X_{\mathrm{pa}}$ et $\mathrm{X}_{\mathrm{f}}$ sont les teneurs respectives de $\mathrm{X}$ dans la phase aqueuse et le fromage $\left(\mathrm{g} \cdot \mathrm{kg}^{-1}\right)$.

\subsection{Analyses statistiques}

Pour étudier l'effet des facteurs « état du fromage » (congelé ou non avant pressage), « stade d'affinage » et « série d'emmental » sur l'extraction des phases aqueuses et lipidiques (vitesse initiale d'écoulement et rendement), une analyse de variance a été effectuée sur l'ensemble des données ( $n=51)$ avec la procédure GLM de SAS [43]. Les moyennes ont été comparées par le test des plus petites différences significatives.

\section{RÉSULTATS ET DISCUSSION}

La composition globale de l'ensemble des fromages étudiés était conforme aux données de la littérature sur l'emmental : extrait sec de 610 à $636 \mathrm{~g} \cdot \mathrm{kg}^{-1}$, matière grasse

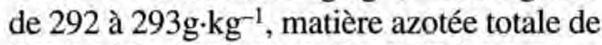
277 à $291 \mathrm{~g} \cdot \mathrm{kg}^{-1}$, Ca de 9,8 à $10,4 \mathrm{~g} \cdot \mathrm{kg}^{-1}$.

\subsection{Dynamique des populations bactériennes}

Les dénombrements effectués dans deux secteurs opposés de la meule présentaient une excellente répétabilité pour les trois flores étudiées : l'écart type de répétabilité s'élevait à $0,13 \log \left(\mathrm{ufc}_{\mathrm{f}} \mathrm{g}^{-1}\right)$, ce qui correspond approximativement à la taille des symboles représentés figure 1 .

\subsection{1. Évolution des levains lactiques}

Les lactobacilles thermophiles atteignaient 8,6 à $9,0 \log \left(\mathrm{ufc}_{\mathrm{f}} \mathrm{g}^{-1}\right)$ en fin de pressage, et présentaient ensuite une chute de viabilité, dont l'ampleur et la cinétique variaient considérablement entre les trois séries d'emmentals étudiés. Dans la série A, leur viabilité diminuait de $70,5 \%$ (soit $0,5 \log$ ufc $\cdot \mathrm{g}^{-1}$ ) pendant le saumurage puis restait stable tout au long de l'affinage. Dans la série B, leur viabilité chutait de $99,8 \%$ (soit 2,6 log) en cave tempérée. Enfin, dans la série $C$, la viabilité diminuait peu en cave tempérée $(0,3 \log )$ mais chutait rapidement dès l'entrée en cave chaude, conduisant à $99,9 \%$ (soit 3,3 log) de mortalité en sortie de cave chaude (figure 1). Seule une chute rapide de viabilité dès le début de l'affinage (cas de la série B), avait été décrite pour cette flore [13, 14]. Ces différences n'ont pu être reliées à des différences de développement des flores d'affinage. En effet par exemple, dans les séries A et B, la viabilité des lactobacilles évoluait de manière différente alors que le développement des flores d'affinage y était très similaire. 

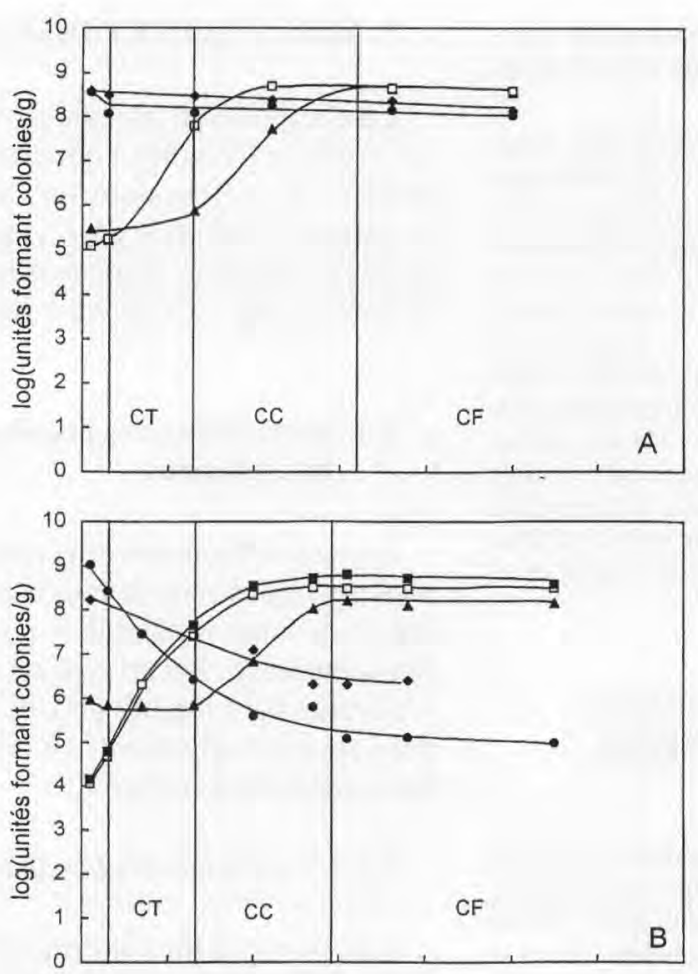

Figure 1. Évolution des levains lactiques et propioniques et de la flore lactique non levain durant l'affinage de trois séries d'emmentals de l'Ouest de la France fabriqués à partir de lait thermisé. Stades d'affinage : CT : cave tempérée ; $\mathrm{CC}$ : cave chaude ; CF : cave froide. Flores : - lactobacilles thermophiles ; streptocoques thermophiles ; $\boldsymbol{\Delta}$ bactéries propioniques; bactéries lactiques non levains dénombrées sur :

MRS incubé à $15^{\circ} \mathrm{C}$ et sur

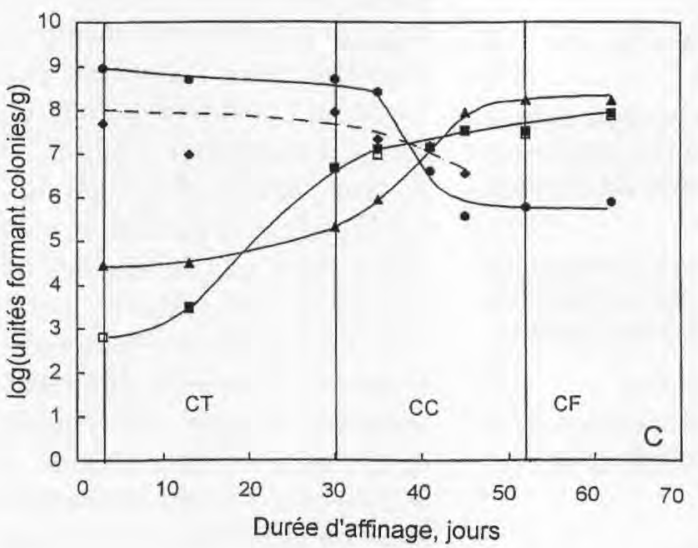
$\square \mathrm{FH}$.

Figure 1. Evolution of lactic and propionic starters and non-starter lactic acid bacteria throughout ripening in three thermised milk Emmental cheeses from the West of France. Ripening steps: CT: temperate room; CC: warm room; CF: cold room. Flora: - thermophilic lactobacilli; - thermophilic streptococci; $\Delta$ propionibacteria; non-starter lactic acid bacteria enumarated on: MRS incubated at $15^{\circ} \mathrm{C}$ and on: $\square \mathrm{FH}$.

L'aptitude d'un micro-organisme à rester viable malgré l'épuisement de son substrat carboné est probablement un caractère très dépendant des souches, tout comme l'aptitude à s'autolyser [35]. Nous n'avons cependant pas vérifié, dans le cadre de cette étude, si les différences de viabilité traduisaient des différences d'autolyse, comme cela a été montré pour des lactocoques [12].

Les streptocoques thermophiles subissaient également une chute de viabilité, qui variait de $64 \%$ (série A) à plus de $98 \%$ (série B) (figure 1). Ils étaient mieux détectés par incubation en anaérobiose qu'en 
aérobiose (résultats non présentés) mais la sélectivité du milieu M 17 était cependant insuffisante pour permettre leur détection en fin d'affinage dans les séries $\mathrm{B}$ et $\mathrm{C}$.

\subsection{2. Évolution des levains propioniques}

Les numérations ne différaient que de $0,1 \log$ au maximum sur les milieux LGA et Pal Propiobac. Les bactéries propioniques se développaient essentiellement en cave chaude, avec un temps de génération maximal d'environ $35-42 \mathrm{~h}$, voisin de celui relevé dans des fabrications expérimentales [48]. Malgré une population initiale différente dans les trois séries, leur population finale atteignait 8,0 à $8,6 \log \left(\mathrm{ufc}^{-\mathrm{g}^{-1}}\right)$ (figure 1 ). Ce niveau assez bas par rapport aux valeurs de $9,0 \log \left(\mathrm{ufc} \cdot \mathrm{g}^{-1}\right)$ classiquement rencontrées en fabrication d'emmental $[32,46,48]$ pourrait être partiellement dû, dans le cas des séries $\mathrm{A}$ et $\mathrm{B}$, à l'affinage relativement court de ces fabrications. Dans la série C cependant, de manière surprenante, la cinétique de croissance des bactéries propioniques s'est ralentie avant même la sortie de cave chaude.

\subsection{3. Évolution et identification de la flore non levain}

La flore non levain se développait dès le début de l'affinage pour les trois séries de fromages, avec un temps de génération maximal en cave tempérée d'environ $25 \mathrm{~h}$ à $37 \mathrm{~h}$. Malgré des différences importantes de population initiale (plus de $2 \mathrm{log} \mathrm{ufc} \cdot \mathrm{g}^{-1}$ d'écart en sortie de saumure entre les séries $A$ et $\mathrm{C}$ ), cette flore atteignait au minimum $8,0 \log \left(u f c \cdot g^{-1}\right)$ au cours de l'affinage en cave chaude dans les 3 séries (figure 1). Des niveaux de population équivalents de lactobacilles non levains (entre 8,0 et 8,6 log (ufc $\cdot \mathrm{g}^{-1}$ )) ont été trouvés dans 9 cas sur 10 pour d'autres emmentals affinés représentant les principaux producteurs de l'Ouest de la France (résultats non présentés). Les niveaux de population dénombrés sur MRS $-15^{\circ} \mathrm{C}$ étaient identiques ou légèrement supérieurs à ceux obtenus sur FH (figure 1). Seules des bactéries de type lactobacilles étaient observées sur ces deux milieux (morphologie bacilles ou coccobacilles, Gram positif, catalase négative). Tous les isolats ont été identifiés comme des lactobacilles hétérofermentaires, dont $11 \%$ d'hétérofermentaires stricts. La distribution des espèces était similaire sur les deux milieux, à l'exception de Lactobacillus fermentum, sous-représenté sur le milieu MRS- $15{ }^{\circ} \mathrm{C}$, vraisemblablement parce que cette espèce se développe mal à $15^{\circ} \mathrm{C}$. Sur le milieu FH cependant, tous les isolats strictement hétérofermentaires formaient des colonies atypiques (grises ou translucides, de diamètre inférieur à $0,2 \mathrm{~mm}$ ), ainsi que ceux identifiés comme Lactobacillus curvatus. Lactobacillus paracasei prédominait pendant tout l'affinage pour les deux séries d'emmental étudiées, représentant en moyenne 56 et $88 \%$ des isolats caractérisés respectivement, pour les séries $\mathrm{B}$ et $\mathrm{C}$. Les autres lactobacilles retrouvés étaient $L$. curvatus, $L$. fermentum, L. buchneri pour les deux séries ainsi que L. rhamnosus et $L$. brevis pour la série B. Le nombre d'isolats identifiés est insuffisant pour nous permettre de montrer d'éventuels changements dans la distribution des espèces non levains au cours de l'affinage et, par conséquent, de chute de viabilité pour une espèce donnée.

Les niveaux de population atteints sont comparables à ceux qui ont été observés dans des fromages à pâte pressée cuite fabriqués à partir de lait cru [50, 13]. En revanche, les entérocoques se trouvaient à un niveau inférieur, ne dépassant pas $6,0 \log \left(\mathrm{ufc} \cdot \mathrm{g}^{-1}\right)$ dans la série A et $4,0 \log \left(\mathrm{ufc}_{\mathrm{f}} \mathrm{g}^{-1}\right)$ dans les deux autres séries (données non présentées). La prédominance de l'espèce $L$. paracasei (c'est-à-dire Lactobacillus casei subsp. casei selon Dicks et al. [15], concorde avec les résultats obtenus en technologie Comté [50, 13], ainsi que, plus largement, dans des fromages à pâte pressée fabriqués à partir de lait pasteurisé comme le cheddar $[30,40]$, ou des fromages norvégiens et suédois [34]. 


\subsection{Extraction de la phase aqueuse}

Le pressage permettait d'extraire en moyenne $10 \mathrm{~g}$ de phase aqueuse pour $100 \mathrm{~g}$ fromage, ce qui correspond à près d'un quart de la phase aqueuse contenue dans le fromage, d'après l'équation [1]. En outre, $5,5 \mathrm{~g}$ de phase lipidique étaient également extraits pour $100 \mathrm{~g}$ fromage, soit $19 \%$ de la matière grasse du fromage. Ces rendements moyens recouvraient une variabilité importante d'une extraction à l'autre malgré des conditions standardisées de pressage, comme l'illustrent les cinétiques d'écoulement des phases aqueuses obtenues pour la série $\mathrm{A}$ à partir d'échantillons pressés en frais ou après congélation/décongélation (figure 2). Ainsi, la congélation du fromage multipliait par 2 la vitesse initiale d'écoulement de la phase aqueuse et par 1,5 son rendement mais n'affectait pas l'écoulement de la phase lipidique (tableau II). L'écoulement et le rendement variaient également en fonction du stade d'affinage et de la série d'emmental. Plus les fromages étaient affinés, plus l'écoulement de la phase aqueuse était lent et celui de la phase lipidique rapide (tableau II). Les différences d'écoulement de la phase aqueuse reflètent des différences d'organisation structurale du fromage, organisation qui évolue au cours de l'affinage du fait des modifications de composition et d'éventuels réarrangements moléculaires. L'écoulement de la phase lipidique dépend de l'état des globules gras. La détérioration de leur membrane au cours de l'affinage pourrait faciliter l'extraction de la phase lipidique.

La congélation des échantillons avant pressage ne modifiait ni la teneur en MAT des phases aqueuses, ni leurs teneurs en $\mathrm{P}$, $\mathrm{K}, \mathrm{Cl}$ et $\mathrm{Na}$ mais entraînait une diminution moyenne de $8 \%$ du Ca et de $5 \%$ du $\mathrm{Mg}$ des phases aqueuses (résultats non présentés). Ces différences mineures de composition malgré des cinétiques d'extraction très différentes valident le modèle d'un réseau paracaséinique poreux emprisonnant une phase aqueuse continue, dont l'extractibilité varie avec les changements de structure du réseau qu'induisent la congélation du fromage ou l'affinage.

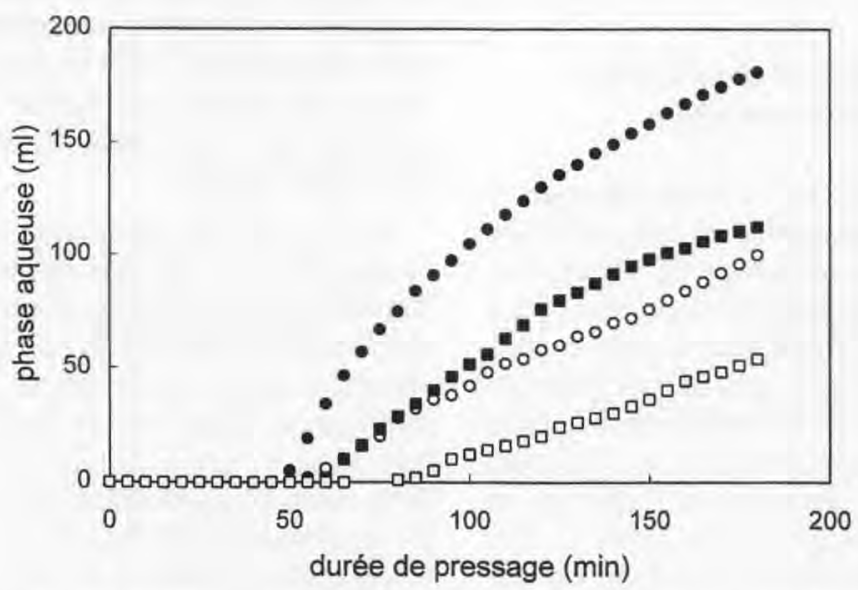

Figure 2. Cinétique d'écoulement de la phase aqueuse de $1800 \mathrm{~g}$ d'emmental extraite par pressage pour des échantillons pressés « en frais " (symboles ouverts) ou après congélation/décongélation (symboles fermés) à 2 stades d'affinage : $O, 0$ : sortie de saumure ; $\square$, : $\mathbf{0}$ : découpe.

Figure 2. Flow kinetic of aqueous phase during extraction by pressing for samples freshly pressed (open symbols) or after freezing/thawing (closed symbols) at 2 ripening stages: $\bigcirc$, end of brining; $\square$, : commercialization. 
Tableau II. Influence de la congélation préalable des échantillons, du stade d'affinage et de la série d'emmental sur la cinétique d'écoulement des phases aqueuses et lipidiques de l'emmental lors de l'extraction par pressage.

Table II. Influence of the freezing of Emmental cheese samples, the ripening stage and the fabrication on the flow kinetic of aqueous phase and oil phase during the extraction by pressing.

\begin{tabular}{|c|c|c|c|c|}
\hline & \multicolumn{2}{|c|}{ Phase aqueuse } & \multicolumn{2}{|c|}{ Phase lipidique } \\
\hline & $\begin{array}{l}\text { Vitessed } \\
(\mathrm{mL} / \mathrm{min})\end{array}$ & $\begin{array}{c}\text { Rendement } \\
(\%)\end{array}$ & $\begin{array}{l}\text { Vitessea } \\
(\mathrm{mL} / \mathrm{min})\end{array}$ & $\begin{array}{c}\text { Rendement } \\
(\%)\end{array}$ \\
\hline \multicolumn{5}{|l|}{ État } \\
\hline frais & $1,4^{\text {as }}$ & $6,8^{\mathrm{a}}$ & $1,3^{\mathrm{a}}$ & $5,3^{\mathrm{a}}$ \\
\hline congelé/décongelé & $2,8^{b}$ & $10,6^{\mathrm{b}}$ & $1,0^{\mathrm{a}}$ & $5,1^{\mathrm{a}}$ \\
\hline \multicolumn{5}{|l|}{ Stade d'affinage* } \\
\hline SP & $3,4^{\mathrm{d}}$ & $11,2^{\mathrm{b}}$ & $0,7^{\mathrm{a}}$ & $3,7^{\mathrm{a}}$ \\
\hline SS & $3,1^{\mathrm{cd}}$ & $12,7^{c}$ & $1,1^{\text {ab }}$ & $4,7^{\mathrm{ab}}$ \\
\hline $\mathrm{ECC}$ & $2,6^{\text {bcd }}$ & $9,7^{\mathrm{a}}$ & $1,3^{\mathrm{b}}$ & $5,6^{\mathrm{bc}}$ \\
\hline MCC & $2,2^{\mathrm{b}}$ & $8,8^{\mathrm{a}}$ & $1,1^{\mathrm{b}}$ & $5,3^{\text {bc }}$ \\
\hline SCC & $2,3^{\mathrm{b}}$ & $9,2^{\mathrm{a}}$ & $1,4^{\mathrm{b}}$ & $6,4^{c}$ \\
\hline $\mathrm{D}$ & $1,6^{\mathrm{a}}$ & $8,8^{\mathrm{a}}$ & $1,4^{b}$ & $6,6^{c}$ \\
\hline \multicolumn{5}{|l|}{ Série d'emmental } \\
\hline A & $1,6^{\mathrm{a}}$ & $7,9^{\mathrm{a}}$ & $0,9^{\mathrm{a}}$ & $4,5^{\mathrm{a}}$ \\
\hline $\mathrm{B}$ & $2,8^{b}$ & $10,9^{\mathrm{b}}$ & $1,7^{b}$ & $6,9^{b}$ \\
\hline $\mathrm{C}$ & $3,1^{\mathrm{b}}$ & $11,0^{\mathrm{b}}$ & $1,0^{\mathrm{a}}$ & $4,7^{\mathrm{a}}$ \\
\hline
\end{tabular}

ă Vitesse initiale d'écoulement, calculée sur 15 à 20 min pour les phases aqueuses, 20 à 30 min pour les phases lipidiques. * Stades d'affinage : voir tableau I. \$ Pour un facteur donné (état de l'échantillon, stade d'affinage ou série d'emmental), les valeurs d'une même colonne portant la même lettre ne diffèrent pas significativement $(P<0,05)$ par le test des plus petites différences significatives.

a Initial flow rate, calculated during 15 to $20 \mathrm{~min}$ for aqueous phases and 20 to $30 \mathrm{~min}$ for oils. * Ripening stages: see table $I .{ }^{5}$ for a given factor (fresh or freezed cheese sample, ripening stage or fabrication), values of a column with the same superseript do not differ $(P<0.05)$ by the least significant difference test.

\section{3. Évolution de la composition de la phase aqueuse au cours de l'affinage}

Les durées des étapes d'affinage différant d'une série d'emmental à l'autre, nous avons choisi de ne pas présenter de résultats «moyens », mais d'illustrer l'évolution de la composition de la phase aqueuse de la série d'emmental $\mathrm{A}$ et de la comparer à celle des séries $\mathrm{B}$ et $\mathrm{C}$.

\subsubsection{Extrait sec, $p H$, sucres et acides organiques}

L'extrait sec de la phase aqueuse doublait au cours de l'affinage entre les stades sortie presse et découpe. Cet accroissement était essentiellement dû à l'apport de $\mathrm{NaCl}$ lors du saumurage et à la protéolyse en cave chaude qui solubilisait dans la phase aqueuse du fromage une partie de sa matière azotée (tableau III, série A). Le pH de la phase aqueuse, augmentait d'environ 0,25 unité au cours de l'affinage (tableau III, série A). Il était légèrement supérieur à celui du fromage (de 0,06 à 0,15 unité $\mathrm{pH}$ selon le stade d'affinage, résultats non montrés), en accord avec les données de Lucey et al. [36].

L'évolution des sucres et acides organiques était similaire pour les trois séries d'emmental et se caractérisait par un épuisement quasi total des sucres dès le stade «sortie presse », une diminution du citrate 
en cave tempérée et une fermentation du lactate en cave chaude (tableau III, série A). Le lactose, le glucose et le galactose n'étaient pas détectables dans les phases aqueuses, sauf en tout début d'affinage pour la phase aqueuse de la série A dans laquelle $0,3 \mathrm{~g} \cdot \mathrm{kg}^{-1}$ de lactose subsistait jusqu'en sortie de saumure. L'ion lactate était majoritairement présent sous la forme $\mathrm{L}(+): 65$, 73 et $57 \%$ respectivement dans les trois séries, ce qui correspond aux proportions habituellement rencontrées lorsque le levain lactique se compose d'un mélange de Streptococcus thermophilus et de Lactobacillus helveticus, éventuellement associés à Lactobacillus delbrueckii subsp. lactis $[8,47]$. Pour les trois séries, les teneurs en lactate ne diminuaient que de 1 à $2 \mathrm{~g} \cdot \mathrm{kg}^{-1}$ de phase aqueuse et les produits de la fermentation propionique restaient très faibles : 1 à $2 \mathrm{~g} \cdot \mathrm{kg}^{-1} \mathrm{~d}^{\prime}$ acide propionique et 2 à $4 \mathrm{~g} \cdot \mathrm{kg}^{-1}$ d'acide acétique. Ces résultats sont cohérents avec le développement limité de la population propionique dans ces fromages. Le citrate des phases aqueuses atteignait, selon la série d'emmental, 1,7 à $2,4 \mathrm{~g} \cdot \mathrm{kg}^{-1}$ en début d'affinage. Il chutait ensuite en cave tempérée et en début en cave chaude à des teneurs inférieures à $0,4 \mathrm{~g} \cdot \mathrm{kg}^{-1}$. Sa diminution coïncide avec le développement des lactobacilles hétérofermentaires. Parmi ceuxci, certaines espèces, telles que Lactobacillus casei et Lactobacillus rhamnosus, fermentent le citrate du fromage en acétate, formate et $\mathrm{CO}_{2}$ [29]. Cette fermentation expliquerait environ la moitié de l'acétate présent dans l'emmental avant que ne débute la fermentation propionique.

\subsubsection{Matières azotées}

Plus d'un quart de la matière azotée présente en fin d'affinage dans la phase aqueuse était libéré pendant le premier jour de fabrication. Ainsi, dès le stade "sortie presse", la matière azotée de la phase aqueuse s'élevait à 13 à $18 \mathrm{~g} \cdot \mathrm{kg}^{-1}$, dont 2 à $5 \mathrm{~g} \cdot \mathrm{kg}^{-1}$ d'acides aminés libres. La présence d'une quantité appréciable d'acides aminés libres dans des emmentals finlandais d'un jour, signalée dès 1953 par Kiuru et al. [31], illustre le rôle du potentiel peptidasique des lactobacilles thermophiles.

Tableau III. Évolution de la composition de la phase aqueuse d'un emmental de l'Ouest de la France (série A) au cours de l'affinage, en $\mathrm{g} \cdot \mathrm{kg}^{-1}$.

Table III. Evolution of the compostion of the aqueous phase of an Emmental cheese from the West of France (fabrication A) throughout ripening, in $\mathrm{g} \cdot \mathrm{kg}^{-1}$.

\begin{tabular}{lccccc}
\hline Stades d'affinage* & SP & SS & ECC & MCC & SCC \\
\hline $\mathrm{pH}$ & 5,24 & 5,30 & 5,38 & 5,44 & 5,48 \\
Extrait sec & 71,7 & 89,4 & 95,9 & 118,4 & 139,6 \\
Matière azoté & 18,6 & 18,0 & 23,8 & 46,1 & 68,1 \\
Lactate & 34,1 & 34,1 & 34,6 & 32,4 & 32,3 \\
Citrate & 2,1 & 2,4 & 1,7 & 0,2 & nd \\
Acétate & nd & nd & 0,5 & 2,9 & 4,0 \\
Propionate & nd & nd & nd & nd & 1,8 \\
Cendres & 19,3 & 33,9 & 35,2 & 40,5 & 38,4 \\
$\mathrm{Na}$ & 0,8 & 7,2 & 7,3 & 8,6 & 8,1 \\
$\mathrm{Cl}$ & 0,8 & 9,7 & 9,1 & 11,2 & 10,8 \\
\hline
\end{tabular}

* Stades d'affinage : voir tableau I ; nd : non détectable.

* Ripening stages: see table 1 ; nd: not detectable. 


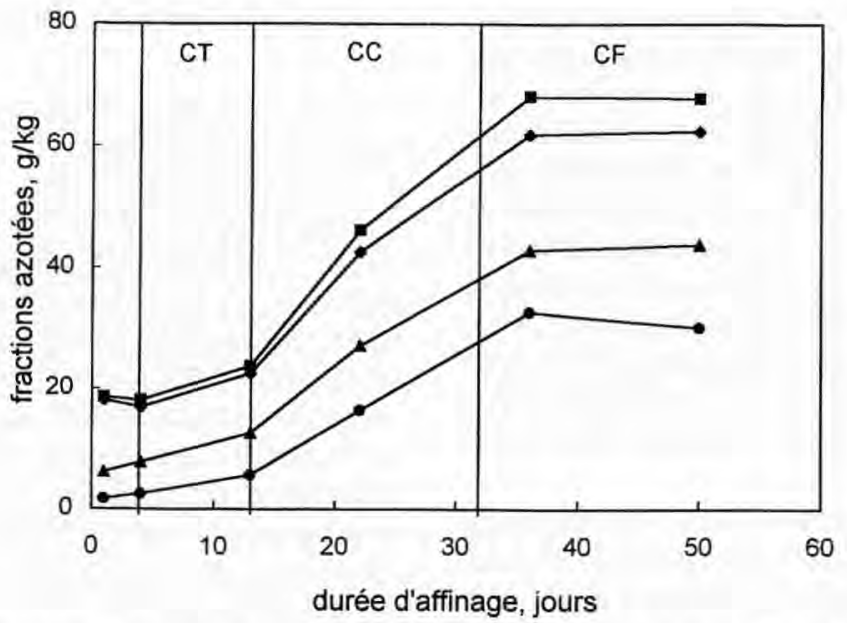

Figure 3. Évolution des fractions azotées de la phase aqueuse durant l'affinage d'un emmental de l'Ouest de la France (série A). Matière azotée : totale; soluble à pH 4,6; $\boldsymbol{\Delta}$ soluble dans l'acide tricholoacétique $12 \%$; acides aminés libres. Stades d'affinage : CT : cave tempérée ; CC : cave chaude ; $\mathrm{CF}$ : cave froide.

Figure 3. Evolution of nitrogen matter in aqueous phases throughout the ripening of three thermised milk-Emmental cheeses from the West of France. total $\mathrm{N}$; $\mathbf{p H} 4$.6-soluble $\mathrm{N}$; $\boldsymbol{\Delta}$ trichloroacetic acid $12 \%$-soluble N; free amino acids. Ripening stages: CT: temperate room; CC: warm room; CF: cold room.

Au cours de l'affinage, la matière azotée des phases aqueuses était multipliée par un facteur 3,4 à 4,2 . Cette augmentation recouvrait des évolutions différentes selon les fractions azotées considérées (figure 3, série A). Ainsi, les protéines précipitables à $\mathrm{pH} 4,6$ (fraction NT - NCN) étaient à peine quantifiables en début d'affinage, et ne dépassaient pas $9 \%$ de la matière azotée de la phase aqueuse en fin d'affinage. La fraction des « gros » peptides et des peptides hydrophobes (fraction NCN - NPN), qui formait 40 à $50 \%$ de la matière azotée totale de la phase aqueuse en sortie de saumure, ne représentait plus que 20 à $30 \%$ en fin d'affinage. La fraction des petits peptides et des peptides hydrophiles (fraction NPN - AAL) présentait un accroissement de 6 à $14 \mathrm{~g} \cdot \mathrm{kg}^{-1}$ entre la sortie saumure et la découpe mais sa proportion relative par rapport à la matière azotée totale variait peu (20 à $30 \%$ selon la série). Les acides aminés libres formaient la fraction azotée qui augmentait le plus dans la phase aqueuse au cours de l'affinage, avec un accroissement de 22 à $28 \mathrm{~g} \cdot \mathrm{kg}^{-1}$ entre la sortie saumure et la découpe et représentaient 44 à $47 \%$ de la matière azotée des phases aqueuses en fin d'affinage pour les 3 séries.

La principale augmentation de la matière azotée des phases aqueuses avait lieu en cave chaude (figure 3, série A). Ainsi, on observait une accélération de la libération de l'ensemble des fractions azotées dès l'entrée en cave chaude, plus marquée pour la série A dans laquelle la teneur en NT de la phase aqueuse augmentait 4 fois plus vite en cave chaude qu'en cave tempérée, contre environ 2 fois pour les séries B et C. Ces différences entre séries sont vraisemblablement très liées aux températures des caves tempérée et chaude, qui différaient entre les séries. La proportion de matière azotée libérée dans la phase aqueuse au cours de l'affi- 
nage en cave chaude représentait, selon les séries, entre 37 et $65 \%$ de la quantité présente en fin d'affinage, celle des acides aminés libres entre 35 et $82 \%$. L'accélération de la protéolyse avait lieu bien avant que la population des bactéries propioniques ait atteint un niveau leur permettant d'exercer une influence quantifiable sur la protéolyse, il est donc légitime de penser que ce groupe bactérien n'a pas de rôle majeur dans le phénomène de protéolyse, tout au moins pour ce type de fabrication à affinage relativement court.

La composition en acides aminés totaux de la phase aqueuse variait peu au cours de l'affinage. Elle était globalement proche de celle du fromage, avec cependant quelques différences, liées notamment à la présence des protéines sériques : ainsi, la lysine et la cystéine étaient plus représentées dans les phases aqueuses. À l'inverse, les proportions de tyrosine, phénylalanine, proline et méthionine étaient inférieures à celle de l'emmental pour les trois séries étudiées (figure 4, série A).

\subsubsection{Acides aminés libres}

Tous les acides aminés libres augmentaient dans les phases aqueuses, à l'exception de l'arginine pour les 3 séries d'emmental et de l'acide aspartique et l'histidine dans la série $\mathrm{C}$, dont les teneurs diminuaient en cave chaude. La phase aqueuse présentait l'avantage de permettre la quantification des acides aminés libres dès le début de l'affinage, y compris les acides aminés minoritaires tels que la tyrosine, l'isoleucine, la glycine ou la méthionine, dont la détection est limitée en début d'affinage par le facteur de dilution du fromage qu'impose une méthode classique d'extraction.

Le profil des acides aminés libres, exprimé en pourcentage relatif de chaque acide aminé, variait globalement peu au cours de l'affinage (figure 5, série A). Ainsi, les 4 acides aminés principaux de la caséine, acide glutamique, leucine, lysine et proline représentaient durant tout l'affinage 40 à $53 \%$ des acides aminés libres pour les

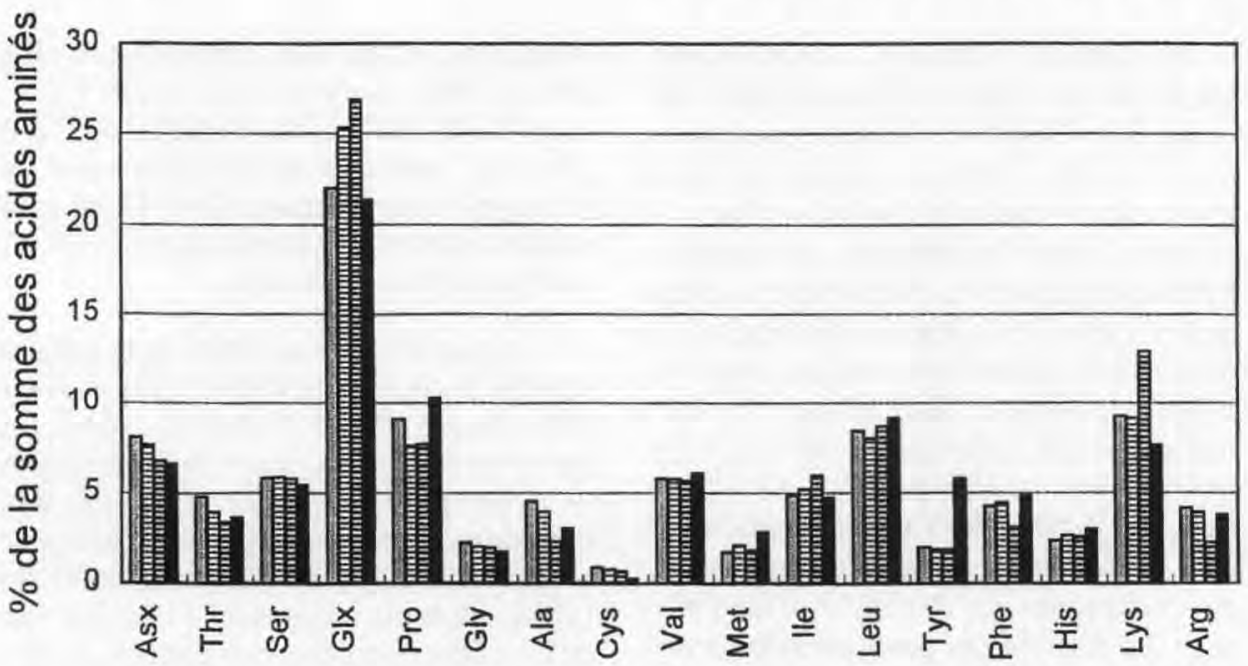

Figure 4. Profil des acides aminés totaux de la phase aqueuse d'un emmental de l'Ouest de la France (série A) à 3 stades d'affinage : 国 sortie presse ; 目entrée cave chaude ; 目 découpe et $\mathbf{p}$ profil des acides aminés totaux du fromage entier (sortie presse).

Figure 4. Total amino acids distribution in the aqueous phase of an Emmental cheese from the West of France (fabrication A) at 3 ripening stages: 国 after the press; 目 before the warm room; $⿴$ a commercialization and: in the entire cheese. 
trois séries d'emmental (et 58 à $67 \%$ si l'on $\mathrm{y}$ ajoute la valine et l'asparagine). La même observation a été effectuée pour le Cheddar dans lequel le profil des acides aminés libres variait peu entre 1 jour et 6 mois d'affinage malgré une multiplication par un facteur 10 de la teneur en acides aminés libres [28].

Quelques acides aminés voyaient cependant leur proportion varier au cours de l'affinage, ce qui traduit soit leur libération préférentielle à un stade d'affinage, soit leur dégradation ultérieure (figure 5). La comparaison des profils des acides aminés libres et totaux (figures 4 et 5 , série $\mathrm{A}$ ) permet de repérer les acides aminés préférentiellement libérés, c'est-à-dire ceux dont la proportion parmi les acides aminés libres est supérieure à la proportion dans la matière azotée de la phase aqueuse. Ainsi par exemple, l'alanine constituait $12,4 \%$ des acides aminés libres dans la phase aqueuse en sortie de presse, contre seulement $4,5 \%$ des acides aminés totaux. Ce phénomène s'atténuait au cours de l'affinage (figure 4) et était observé dans les trois séries d'emmental. De la même façon, l'arginine (si l'on tient compte de ses produits de conversion, Cit et Orn) ainsi que dans une moindre mesure, Pro, His et Lys, étaient préférentiellement libérées en début d'affinage dans les trois séries d'emmental. En fin d'affinage, d'autres acides aminés libres étaient préférentiellement libérés : Leu, Met dans les trois séries, Gly, Val (séries A et B) et Glu et Asp (série C). À l'inverse, Cys, Thr, Ser, Ile et Tyr étaient proportionnellement moins libérés que la moyenne des acides aminés durant tout l'affinage pour les trois séries d'emmental.

La diminution de l'arginine coïncidait avec l'augmentation de la citrulline et de l'ornithine (figure 5, série A), sa dégradation étant plus rapide que sa libération par les peptidases. D'après les teneurs en ces 3 acides aminés, nous avons calculé que la quantité d'arginine dégradée était comprise entre 0,9 (série C) et $2,5 \mathrm{~g} \cdot \mathrm{kg}^{-1}$ (série A), soit encore 80 à $100 \%$ de l'arginine libre. La dégradation d'arginine par la voie de l'argi-

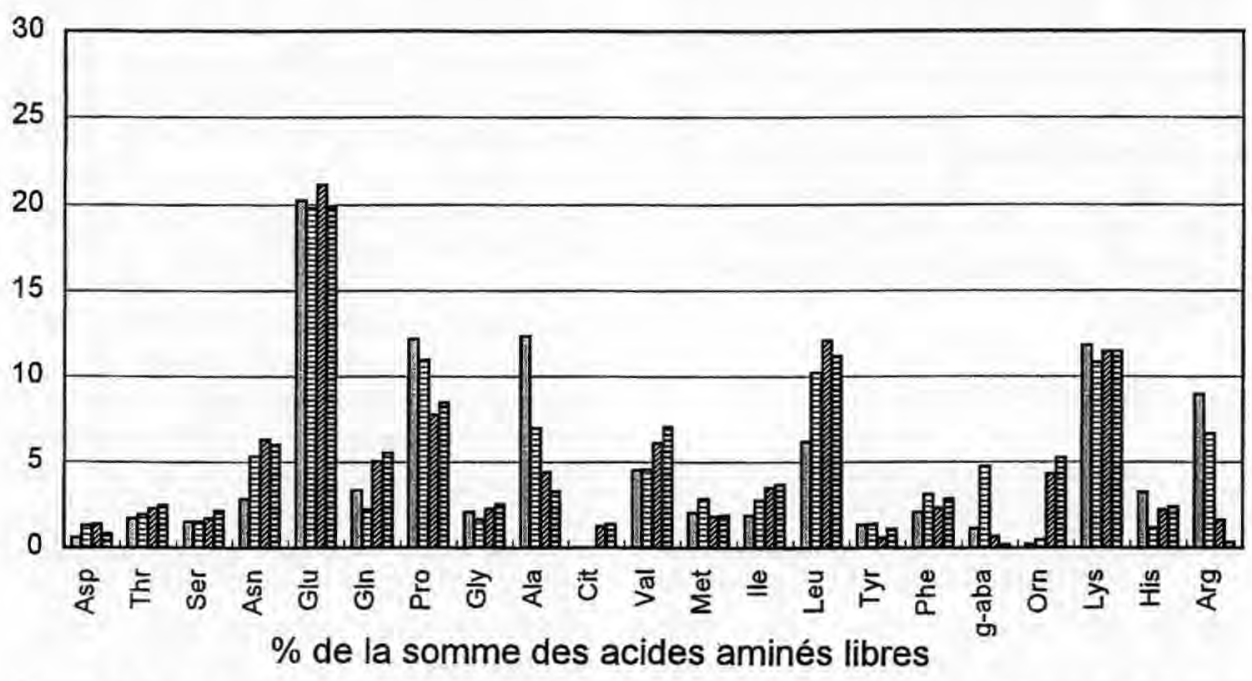

Figure 5. Profil des acides aminés libres de la phase aqueuse d'un emmental de l'Ouest de la France (série A) à 4 stades d'affinage : $⿴ 囗 0$ sortie presse ; 目 entrée cave chaude ; milieu cave chaude ; 目 découpe. g-aba : acide $\gamma$-aminobutyrique.

Figure 5. Free amino acids distribution in the aqueous phase of an Emmental cheese from the West of France (fabrication A) at 3 ripening stages: $⿴$ after the press; 1 before the warm room; mid-

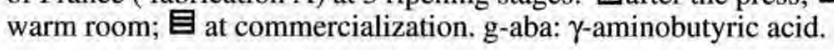


nine déiminase (catabolisme de l'arginine en citrulline $+\mathrm{NH}_{3}$ puis dégradation de la citrulline en ornithine et carbamoyl phosphate) est effectuée par $S$. thermophilus ainsi que par de nombreux lactobacilles hétérofermentaires stricts tels que $L$. brevis, $L$. fermentum et $L$. buchneri [37], retrouvés dans la flore non levain. La dégradation de l'arginine en citrulline a également été mise en évidence chez $L$. helveticus. Pour les séries $\mathrm{A}$ et $\mathrm{B}$, la confrontation des cinétiques biochimiques et microbiologiques nous laisse penser que la flore non levain était à l'origine de la production d'ornithine. La dégradation de l'arginine était décelée lorsque la flore non levain atteignait 7,7 à $8,0 \mathrm{log}$ (ufc $\mathrm{g}^{-1}$ ) et se poursuivait après que cette flore ait atteint sa population maximale. Dans la série $C$, la conversion de l'arginine en citrulline débutait alors que la flore non levain était à un niveau encore assez faible $\left(<6,7 \log \left(u f c \cdot g^{-1}\right)\right)$, la citrulline demeurant le produit majoritaire, ce qui indiquerait plutôt une activité des levains lactiques thermophiles. Un autre acide aminé absent des caséines, 1 'acide $\gamma$-aminobutyrique, provenant de la décarboxylation de l'acide glutamique, était également détecté à des niveaux variables selon la série d'emmental. La présence d'acide $\gamma$-aminobutyrique a été observée dans différents fromages (Camembert, Grana) et a été mise en relation avec l'activité de certains levains lactiques dans le Gouda [51].

\subsubsection{Minéraux}

L'essentiel des échanges minéraux entre le réseau paracaséinique et la phase aqueuse s'effectuait avant même le saumurage, au cours de l'acidification qui, en technologie pâte pressée cuite, a lieu sous presse après l'élimination quasi totale du lactosérum. Ainsi, dès le stade « sortie presse », les teneurs de la phase aqueuse en $\mathrm{Ca}, \mathrm{Mg}$ et $\mathrm{P}$ étaient respectivement 16,10 et 3 fois plus élevées que dans la phase aqueuse du lait. Les cations monovalents $(\mathrm{Na}, \mathrm{K})$ étaient en concentration légèrement supérieure à celle du lait (+ 50 à $80 \%$ ), ce qui indique qu' une fraction de ces minéraux n'était pas soluble dans la phase aqueuse du lait.

Après saumurage, la phase aqueuse s'enrichissait de 7 à $10 \mathrm{~g} \cdot \mathrm{kg}^{-1}$ de $\mathrm{Na}$ et de 8 à $13 \mathrm{~g} \cdot \mathrm{kg}^{-1} \mathrm{de} \mathrm{Cl}$, selon les séries (tableau III, série A). Une augmentation artéfactuelle était constatée entre les stades " sortie saumure » et « milieu cave chaude », due à la combinaison de deux phénomènes : d'une part, le sel incorporé lors du saumurage migrait de la périphérie vers le centre de la meule et d'autre part, les secteurs utilisés pour le pressage subissaient un écroûtage qui éliminait 0,3 à $0,5 \mathrm{~cm}$ en périphérie.

La composition minérale de la phase aqueuse, mis à part $\mathrm{Na}$ et $\mathrm{Cl}$, variait globalement peu au cours de 1'affinage. Les teneurs en $\mathrm{Ca}, \mathrm{Mg}$ et $\mathrm{P}$ présentaient cependant des variations faibles, mais significatives $(p<0,05)$, similaires pour les 3 séries d'emmental. Les teneurs en $\mathrm{Ca}$ et $\mathrm{Mg}$ chutaient respectivement de $0,3 \mathrm{~g} \cdot \mathrm{kg}^{-1}$ et $0,03 \mathrm{~g} \cdot \mathrm{kg}^{-1}$ en moyenne pendant le saumurage puis augmentaient de 0,5 et $0,05 \mathrm{~g} \cdot \mathrm{kg}^{-1}$ au cours de l'affinage (figure 6, série A). La teneur en $\mathrm{P}$ total diminuait de $0,4 \mathrm{~g} \cdot \mathrm{kg}^{-1}$ en moyenne jusqu'en entrée de cave chaude, puis augmentait entre les stades « entrée cave chaude » et « découpe », de manière plus prononcée pour la série $\mathrm{A}\left(+0,4 \mathrm{~g} \cdot \mathrm{kg}^{-1}\right)$ que pour les 2 autres séries $\left(+0,2 \mathrm{~g} \cdot \mathrm{kg}^{-1}\right)$. Les analyses de $\mathrm{P}_{\mathrm{i}}$ effectuées pour la série $\mathrm{A}$ montraient sa diminution durant tout l'affinage et l'augmentation du phosphore organique en cave chaude, parallèlement à la protéolyse (figure 6). La proportion de phosphore organique, quel que soit le stade d'affinage, était comprise entre 12 et $15 \mathrm{mg}$ par g de matière azotée. Cette proportion, presque double de celle des caséines, traduit l'enrichissement de la phase aqueuse en phosphopeptides. $\mathrm{P}_{\mathrm{j}}$ représentait ainsi $80 \%$ du P total au stade « sortie presse " contre $34 \%$ seulement en sortie de cave chaude. La teneur en $\mathrm{K}$, égale à $2,3 \pm$ $0,1 \mathrm{~g} \cdot \mathrm{kg}^{-1}$ en moyenne, ne présentait pas de variations significatives au cours de l'affinage, pour aucune des trois séries. 


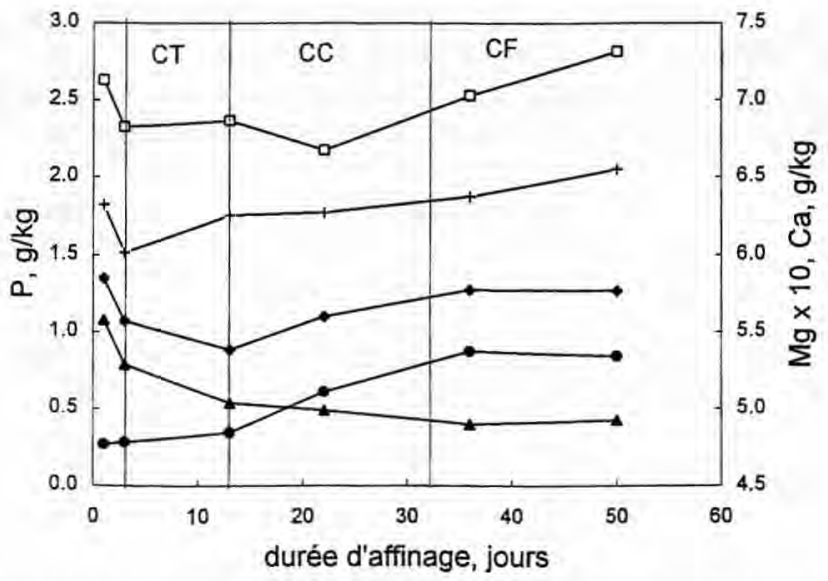

Figure 6. Évolution du calcium $(\square)$, du magnésium (+), du phosphore total $(\bullet)$, du phosphore organique (-) du phosphore minéral $(\boldsymbol{\Delta})$ au cours de l'affinage dans la phase aqueuse d'un emmental de l'Ouest de la France (série A).

Figure 6. Evolution of calcium $(\square)$, magnesium $(+)$, total phosphorus $(\bullet)$, organic phosphorus $(\bullet)$ and inorganic phosphorus $(\mathbf{A})$ in an Emmental cheese from the West of France (fabrication A) throughout ripening.

\subsection{Comparaison des phases aqueuses de différents fromages affinés}

Les phases aqueuses des 3 séries d'emmental avaient une composition très voisine et se distinguaient nettement des phases aqueuses analysées à titre comparatif pour 2 fromages à affinage plus long (emmental Grand Cru et Comté) par une fermentation propionique beaucoup moins prononcée et par une protéolyse plus limitée (tableau IV). L'affinage en cave chaude des emmentals étudiés a cependant pu être écourté du fait de risques de fermentation butyrique, accrus en hiver. Un emmental de même provenance étudié précédemment était néanmoins plus typique [42].

Les teneurs de l'emmental Ouest en lactate résiduel (11,0 à $12,9 \mathrm{~g} \cdot \mathrm{kg}^{-1}$ de fromage) étaient très supérieures aux valeurs habituellement reportées dans la littérature sur l'emmental $\left(7,9 \pm 0,6 \mathrm{~g} \cdot \mathrm{kg}^{-1},[5] ; 3,1 \pm\right.$ $2,0 \mathrm{~g} \cdot \mathrm{kg}^{-1},[47]$ et sont cohérentes avec les faibles teneurs des fromages en propionate ( 0,6 à $1,0 \mathrm{~g} \cdot \mathrm{kg}^{-1}$ de fromage) et en acétate $\left(1,4\right.$ à $\left.1,9 \mathrm{~g} \cdot \mathrm{kg}^{-1}\right)$. En conséquence, le rapport molaire propionate/acétate dans l'emmental Ouest était largement inférieur aux données publiées pour l'emmental : $0,7: 1$ à $1,6: 1$ [11], 0,9:1 à 6:1 [9] alors que les échantillons d'emmental Grand Cru et de Comté se trouvaient dans ces fourchettes.

Les teneurs en matière azotée soluble et en acides aminés libres dans la phase aqueuse des trois séries d'emmental étaient voisines et étaient 2 à 3 fois inférieures à celles de l'emmental Grand Cru et du Comté (tableau IV). La protéolyse des trois séries d'emmental n'était pas corrélée à la chute de viabilité des lactobacilles thermophiles, ce qui peut être interprété de plusieurs façons. Tout d'abord, la chute de viabilité observée ne s'accompagne pas systématiquement d'une lyse des cellules (et ne permet donc pas forcément la libération des peptidases intracellulaires). Même dans le cas d'une lyse, il est possible que malgré les différences observées d'une série à l'autre (figure 1), l'écart entre $70 \%$ de chute de viabilité (série A) et $99 \%$ ou plus (séries B et C) soit difficilement perceptible sur le plan biochimique. Enfin, il est également probable que 
Tableau IV. Comparaison de la composition des phases aqueuses d'un emmental de l'Ouest de la France, d'un emmental Grand Cru et d'un Comté (stade découpe).

Table IV. Comparison of the composition of the aqueous phases of an Emmental from the West of France, an Emmental 'Grand cru' and a 'Comté' cheese at the end of ripening.

\begin{tabular}{|c|c|c|c|c|c|c|}
\hline \multirow[b]{2}{*}{$\left(\mathrm{g} \cdot \mathrm{kg}^{-1}\right)$} & \multicolumn{4}{|c|}{ Emmental Ouest } & \multirow{2}{*}{$\begin{array}{l}\text { Emmental } \\
\text { Grand Cru }\end{array}$} & \multirow[t]{2}{*}{ Comté } \\
\hline & A & B & $\mathrm{C}$ & {$[42]$} & & \\
\hline $\mathrm{pH}$ & 5,50 & 5,58 & 5,45 & 5,80 & 5,58 & 5,67 \\
\hline Extrait sec & 143 & 137 & 125 & 170 & 215 & 260 \\
\hline Matière azotée & 68 & 62 & 54 & 100 & 155 & 198 \\
\hline Acides aminés libres & 31 & 28 & 25 & 56 & 39 & 144 \\
\hline Lactate & 32,6 & 36,3 & 33,4 & 31,8 & 17,0 & 19,5 \\
\hline Acétate & 4,1 & 3,3 & 2,2 & 5,0 & 5,5 & 4,2 \\
\hline Propionate & 2,1 & 1,4 & 1,0 & 4,1 & 8,1 & 4,9 \\
\hline Propionate/acétated & 0,4 & 0,3 & 0,4 & 0,7 & 1,2 & 0,9 \\
\hline $\mathrm{Na}+\mathrm{Cl}$ & 20,6 & 18,0 & 20,2 & 13,6 & 9,9 & 16,3 \\
\hline $\mathrm{Ca}$ & 7,3 & 7,6 & 6,4 & 7,9 & 7,7 & 8,1 \\
\hline K & 2,3 & 2,5 & 2,2 & 2,3 & 2,4 & 2,6 \\
\hline $\mathrm{Mg}$ & 0,66 & 0,70 & 0,62 & 0,66 & 0,70 & 0,74 \\
\hline$P$ total & 1,3 & 1,1 & 1,2 & 1,45 & 2,3 & 3,1 \\
\hline $\mathrm{P}$ organique & 0,9 & - & - & - & 2,1 & 2,6 \\
\hline
\end{tabular}

a Rapport molaire ; - : non déterminé.

Molar ratio; - : not determined.

les levains lactiques utilisés possédaient des potentiels peptidasiques différents ou que l'activité de la flore non levain ait masqué l'activité des levains.

Les teneurs en NT des phases aqueuses étaient bien corrélées à celles du $\mathrm{P}(r=0,98)$, avec un rapport de $1,3 \mathrm{~g}$ de $\mathrm{P}$ pour $100 \mathrm{~g}$ de la matière azotée. Elles étaient également assez bien corrélées aux teneurs en Ca et $\mathrm{Mg}(r=0,70$ et 0,79 , respectivement $)$ (tableau IV). Le pourcentage NS/NT de l'emmental Ouest, déterminé afin d'établir des comparaisons avec les données de la littérature, était de 10 à $11 \%$, valeurs très inférieures aux données publiées : $21 \% \pm 4 \%$ pour l'emmental français [5], $25 \%$ pour l'emmental suisse [47]. L'emmental Grand Cru, avec une valeur de NS/NT de $21 \%$, se situait dans la limite basse donnée par Berdagué et al. [5] pour ce type d'emmental.
Les teneurs en acides aminés libres variaient d'un facteur 2 à 5 (tableau IV) mais les profils des acides aminés libres, exprimés en pourcentage relatif, montraient de grandes similitudes pour l'emmental Ouest, l'emmental Grand Cru, le Comté et l'emmental suisse (figure 7). Cette observation traduit un phénomène de protéolyse assez similaire dans ces différents fromages à pâte pressée cuite. Ainsi, la somme (Glu + Leu + Lys + Pro) représente pour les fromages de cette étude comme pour d'autres emmentals, autour de $50 \%$ des acides aminés libres de l'emmental $[1,47]$. La teneur en proline libre de l'emmental est considérée comme plus élevée que dans d'autres fromages [32]. En proportion relative, elle ne représentait que 7 à $9 \%$ des acides aminés libres dans tous les fromages de notre étude, comme dans l'emmental suisse [47] alors que la teneur en proline des protéines du fromage est de $10 \%$ 
environ. Sa proportion est supérieure ( 9 à $10 \%$ des acides aminés libres) dans les fromages suisses tels que Gruyère, Appenzeller, Sbrinz, Tilsit $[47,33]$ et inférieure (seulement 2 à $7 \%$ des acides aminés libres) dans des pâtes pressées comme l'Edam, le Gouda ou le Cheddar [1, 22]. Sa teneur n'est donc pas liée à la fermentation propionique mais à la technologie de fabrication des pâtes pressées cuites, qui induit une dégradation de la caséine $\beta$ très riche en proline, en favorisant notamment l'activité de la plasmine. La teneur en proline est élevée également dans les fromages de type Bleu [22] dans lesquels la caséine $\beta$ est de même très dégradée [25]. Par ailleurs, les teneurs de nos fromages en phénylalanine ( 3 à $4 \%$ des acides aminés libres) étaient inférieures aux données sur l'emmental finlandais ( 7 à $8 \%$ selon Antila et Antila [1]) et suisse (7,4\% selon Steffen et al. [47]).
Les acides aminés pour lesquels les différences étaient les plus élevées d'un fromage à l'autre étaient ceux qui sont dégradés par les bactéries ou ceux qui résultent du catabolisme bactérien : Arg, Orn, Cit, Asp, Asn, Glu, Gln et acide $\gamma$-aminobutyrique, (figure 7). Ce sont aussi les acides aminés pour lesquels la corrélation avec l'âge du fromage est la moins bonne [7]. Les teneurs les plus faibles en acide aspartique et en asparagine étaient observées pour l'emmental Grand Cru, qui présentait également la fermentation propionique la plus marquée. Cela peut être expliqué par l'activité des bactéries propioniques, qui métabolisent l'acide aspartique en acide fumarique et $\mathrm{NH}_{3}$ [10]. La proportion d'acide aspartique parmi les acides aminés libres est plus faible dans l'emmental que dans d'autres pâtes pressées cuites $[1,47]$. En revanche, la proportion d'asparagine parmi

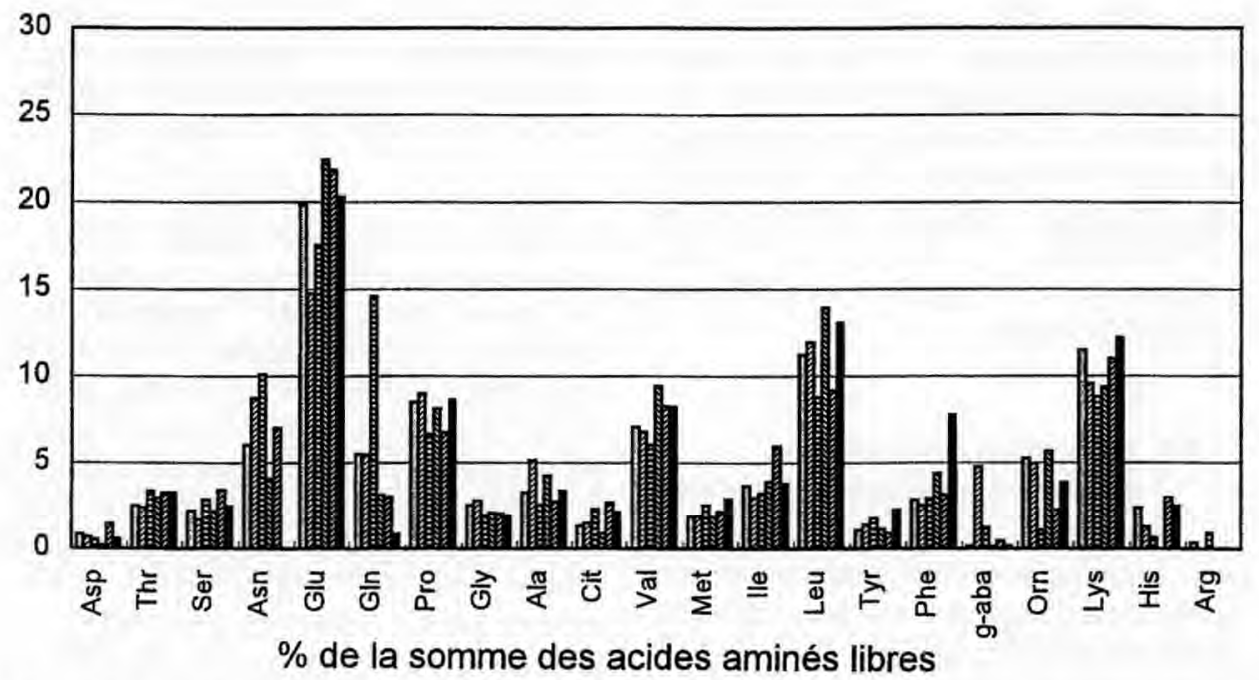

Figure 7. Profil des acides aminés libres de la phase aqueuse de trois emmentals de l'Ouest de la

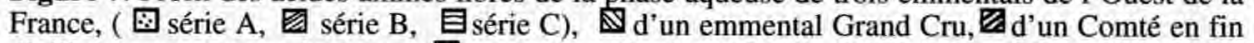
d'affinage, en comparaison avec un emmental suisse (d'après Steffen et al. [47]). g-aba : acide $\gamma$-aminobutyrique

Figure 7. Free amino acids distribution in the aqueous phase of three Emmental cheeses from the West of France (fabrication $\mathrm{A}$, 囷 $\mathrm{B}$ and $\mathrm{O} \mathrm{C}$ ), $\mathrm{Q}$ in an Emmental cheese 'Grand Cru' and in a 'Comté' at the end of ripening, in comparison with Swiss Emmental cheese (from Steffen et al. [47]) g-aba: $\gamma$-aminobutyric acid. 
les acides aminés libres des fromages de notre étude était systématiquement supérieure à celle de l'emmental suisse [7, 47] . La croissance des bactéries propioniques s'accompagne d'une diminution importante de l'acide aspartique mais aussi de l'asparagine (Thierry, données non publiées) mais il est probable qu'elles n'utilisent cette dernière qu'après l'acide aspartique. Pour les autres acides aminés, nous n'avons pu tirer aucune tendance générale à partir des fromages étudiés, les variations entre fromages étant vraisemblablement dues à des variations entre souches de levains et au niveau et à la composition de la flore non levain.

La teneur en $\mathrm{NaCl}$ des phases aqueuses différait également entre les fromages (tableau IV). L'emmental Grand Cru, avec une teneur en $\mathrm{NaCl}$ de $4,8 \mathrm{~g} \cdot \mathrm{kg}^{-1}$ de fromage (calculé d'après la teneur en $\mathrm{Cl}$ ), se trouvait dans la fourchette des données publiées $\left(4,6 \mathrm{~g} \cdot \mathrm{kg}^{-1} \pm 1,6\right)$, tandis que les trois séries d'emmental Ouest étaient légèrement plus salées ( 6 à $10 \mathrm{~g} \cdot \mathrm{kg}^{-1}$ de fromage). La teneur en $\mathrm{Ca}$ et $\mathrm{Mg}$ des phases aqueuses des trois séries d'emmental présentaient des écarts faibles mais significatifs $(p<0,05)$, la série B ayant les teneurs les plus élevées et la série C les teneurs les plus faibles (tableau IV), alors que les teneurs des fromages correspondants ne différaient pas significativement.

\subsection{Proportion des composés du fromage dans la phase aqueuse}

L'augmentation dans la phase aqueuse de certains composés tels que la matière azotée, $\mathrm{Ca}, \mathrm{Mg}, \mathrm{P}$ traduit leur solubilisation dans la phase aqueuse. Leur degré de solubilisation a été évalué selon l'équation [2]. Les résultats étant similaires pour les trois séries d'emmental Ouest, nous n'avons détaillé, comme précédemment, que les valeurs obtenues pour la série A (tableau $\mathrm{V}$ ).

Les proportions de matière azotée solubilisée dans la phase aqueuse restaient infé- rieures à $10 \%$ pour les trois séries d'emmental mais étaient beaucoup plus élevées dans l'emmental Grand Cru et le Comté (tableau $V$ ). Il est à noter que ces proportions étaient systématiquement inférieures aux proportions de matière azotée « soluble $»($ NS/NT) établies par une méthode classique de broyage en tampon citrate, en particulier en début d'affinage (50-60\% du NS retrouvé dans la phase aqueuse, contre $80-90 \%$ en fin d'affinage). Pour les fractions de petits peptides et d'acides aminés, les deux méthodes d'extraction conduisaient à des résultats plus proches : $70 \%$ du NPN obtenu après broyage en tampon en début d'affinage et 90-100\% en fin d'affinage étaient retrouvés dans les phases aqueuses. Par ailleurs, malgré des profils en acides aminés libres globalement similaires, les phases aqueuses se différenciaient légèrement des extraits par broyage par des proportions supérieures en acide glutamique, thréonine et alanine et des proportions inférieures en phénylalanine, tyrosine et histidine (résultats non présentés). L'hydrophobicité des acides aminés pourrait donc jouer un rôle sur leur solubilisation dans la phase aqueuse.

Les proportions du $\mathrm{Mg}$, du Ca et du P solubilisés dans la phase aqueuse différaient fortement pour ces 3 minéraux (tableau $\mathrm{V}$ ). Les valeurs obtenues pour l'emmental Ouest confirment notre précédente étude [42] et sont cohérentes avec les autres données disponibles : 10 à $12 \%$ du $\mathrm{P}$ retrouvé dans la phase aqueuse, d'après les données de Lucey et al. [36]. Dans l'emmental Grand Cru et le Comté, les proportions du Mg échangé entre réseau paracaséinique et phase aqueuse étaient 2 fois plus élevées que celles du $\mathrm{Ca}$ durant tout l'affinage (tableau V), malgré des variations en valeur absolue 10 fois plus faibles pour le $\mathrm{Mg}$ que-pour le $\mathrm{Ca}$ (figure 5). Le Mg était totalement solubilisé dans les fromages à affinage plus long comme l'emmental Grand Cru et le Comté et les proportions de Ca et de $\mathrm{P}$ dans leur phase aqueuse étaient également largement supérieures à celles de l'emmental Ouest (tableau V). 
Tableau V. Fraction des composés du fromage se trouvant dans sa phase aqueuse, d'après l'équation [2]. Table V. Fraction of cheese compouds found in its aqueous phase, calculated with equation [2].

Emmental Ouest (série A)
Emmental Comté Grand Cru

D D

\begin{tabular}{|c|c|c|c|c|c|c|c|c|}
\hline Stades d'affinage* & * SP & SS & ECC & MCC & SCC & D & D & D \\
\hline ESDa & 9,0 & 11,5 & 12,4 & 15,3 & 18,0 & 19,1 & 27,7 & 38,4 \\
\hline Matière azotée & 2,6 & 2,6 & 3,4 & 6,9 & 10,2 & 10,3 & 22,7 & 32.5 \\
\hline Lactate & - & - & - & 96 & 107 & 115 & - & - \\
\hline $\mathrm{Cl}$ & - & 151 & 114 & 112 & 130 & 123 & 86 & 109 \\
\hline K & 103 & 125 & 115 & 119 & 118 & 126 & 133 & 103 \\
\hline $\mathrm{Ca}$ & 30 & 29 & 29 & 29 & 31 & 33 & 34 & 41 \\
\hline $\mathrm{Mg}$ & 70 & 68 & 70 & 73 & 75 & 78 & 123 & 141 \\
\hline $\mathrm{P}$ & 7,9 & 7,1 & 6,6 & 7,6 & 8,8 & 8,9 & 16,6 & 24,1 \\
\hline
\end{tabular}

* Stades d'affinage : voir tableau I. a : extrait sec dégraissé ; - : non déterminé.

* Ripening stages: see table $I$. : : dry matter without fat; - : not determined.

Les proportions calculées dans le tableau $V$ devraient être comprises entre $0 \%$ (composé absent de la phase aqueuse) et $100 \%$ (composé totalement solubilisé). Elles atteignaient cependant des valeurs largement supérieures à $100 \%$ pour certains composés, tels le lactate, le $\mathrm{Cl}$ ou le K (tableau V). Cela peut être expliqué par une sous-estimation du composé dans le fromage, ou par une surestimation de la fraction de phase aqueuse dans le fromage $\mathrm{F}_{\mathrm{pa}}$, ou par une combinaison des deux phénomènes. Le lactate et le $\mathrm{Cl}$ ont vraisemblablement été sous-évalués dans le fromage du fait de l'emplacement du prélèvement des échantillons (mi-rayon et mihauteur) et des gradients décroissants de l'extérieur vers le centre de la meule pour ces deux composés (Steffen, 1975, cité par Moquot, [39]). Les valeurs obtenues pour $\mathrm{K}$ n'ont pu être expliquées que par une erreur liée au dosage de $\mathrm{K}$ dans le fromage à partir des cendres (incinération à $550^{\circ} \mathrm{C}$ ). L'idéal, pour évaluer précisément la part des 2 hypothèses dans l'obtention de proportions supérieures à $100 \%$, serait de disposer d'un composé non chargé, uniformément réparti dans le fromage. Malgré leurs limites et leur surévaluation probable, les proportions établies par cette méthode simple restent comparables entre elles et traduisent le phénomène d'affinage.

Les proportions moyennes d'acétate et de propionate retrouvées dans la phase aqueuse étaient de $112 \% \pm 28 \%$. Dans ce cas, il est possible également que des pertes au cours de l'extraction de ces deux composés très volatils aient conduit à une sousévaluation de leur teneur dans le fromage. Malgré cette possibilité, nos résultats démontrent que ces 2 composés sont quasi totalement dans la phase aqueuse, en accord avec les résultats de Biede et Hammond [6] (97 et $99 \%$ respectivement de l'acétate et du propionate). Les coefficients de partage entre le 1-octanol et l'eau ne sont donc pas applicables aux phases lipidique et aqueuse de l'emmental, comme cela avait été proposé [42].

\section{CONCLUSION}

Cette étude apporte une vision globale des transformations biochimiques au cours de l'affinage de l'emmental et ouvre de nouvelles perspectives de recherche. L'évolution 
la plus marquée est celle de la matière azotée, dont la cinétique d'hydrolyse est très étroitement liée aux températures respectives des caves tempérées et chaudes. Le profil en acides aminés libres varie relativement peu au cours de l'affinage, à l'exception des acides aminés faisant l'objet d'une dégradation microbienne. De même, les profils en acides aminés libres de l'emmental de fabrication industrielle sont très similaires à ceux de fromages voisins de fabrication traditionnelle malgré des différences marquées de leur degré de protéolyse.

L'extraction de la phase aqueuse par pressage présente plusieurs avantages pour suivre les transformations biochimiques du fromage. Elle permet tout d'abord d'évaluer les échanges minéraux entre le réseau paracaséinique et la phase aqueuse au cours de l'affinage. Dans le cas de l'emmental, l'essentiel de ces échanges a lieu lors de l'acidification sous presse mais de légères variations sont observées également pour $\mathrm{Ca}, \mathrm{Mg}$ et $\mathrm{P}$ au cours de l'affinage. Ensuite, l'extraction de la phase aqueuse s'effectue sans dilution, ce qui permet de quantifier certains composés comme les acides aminés libres, même en tout début d'affinage. Enfin, sur un tout autre plan, les données sur la cinétique d'extraction de la phase aqueuse, qui varient beaucoup selon le fromage et le stade d'affinage, pourraient être utilisées comme indicateurs des changements de structure du fromage, ce qui n'a pas été abordé dans le cadre de cette étude. L'extraction de la phase aqueuse est cependant une méthode relativement lourde qui nécessite des échantillons de grande taille. Par ailleurs, bien que la lipolyse soit réduite dans l'emmental, il conviendrait de faire l'analyse des acides gras libres pour compléter l'étude des transformations biochimiques pendant 1 'affinage.

Concernant les flores et leurs activités, nous avons montré que la cinétique de chute de viabilité des levains lactiques thermophiles diffère fortement d'un fromage à l'autre. La signification de ces observations reste à étudier. La disparition du citrate et de l'arginine est très vraisemblablement liée au développement des lactobacilles hétérofermentaires non levains. Cette flore atteint un niveau élevé dès l'entrée en cave chaude et présente une distribution des espèces voisine de celle observée pour des fromages fabriqués au lait cru, ce qui nous conduit à penser que la présence et le niveau atteint dans le fromage par les différentes espèces de lactobacilles non levains dépendent vraisemblablement moins de leur niveau initial respectif dans le lait de fabrication que de leur aptitude à se multiplier à basse température $\left(10-15^{\circ} \mathrm{C}\right)$, en l'absence de lactose et de ses produits d'hydrolyse. Compte tenu du potentiel enzymatique de cette flore [40], elle contribue probablement à d'autres transformations biochimiques de l'affinage. Le développement de la flore propionique ainsi que la fermentation du lactate étaient réduits dans les trois séries d'emmental étudiées.

\section{REMERCIEMENTS}

Nous tenons à remercier C. Brassac, G. Le Gars, M-B. Maillard, Y. Le Graët et M. Piot pour leur contribution aux analyses, A. Pierre et S. Lortal pour leur relecture du manuscrit ainsi que les entreprises qui nous ont fourni les fromages. Ce travail a été cofinancé par l'Institut national de recherche agronomique (AIP Matural) et par la région Bretagne.

\section{RÉFÉRENCES}

[1] Antila V., Antila M., Des Gehalt des finnischen Käses an freien Aminosäuren, Milchwissenschaft 23 (1968) 597-602.

[2] Baird R.M., Corry J.E.L., Curtis G.D.W., Kanamycin Aesculin Azide (KAA) agar, Int. J. Food Microbiol. 5 (1987) 221-222.

[3] Berdagué J.L., Jeunet R., Grappin R,, Affinage et qualité du Gruyère de Comté, III. Fermentation lactique et teneurs en acides gras volatils des fromages de Comté, Lait 67 (1987) 249-263.

[4] Berdagué J.L., Grappin R., Affinage et qualité du Gruyere de Comté, II. Influence de l'affinage sur l'évolution des caractéristiques physico-chimiques des fromages, Lait 67 (1987) 237-247.

[5] Berdagué J.L., Grappin R., Delacroix-Buchet A., Chaillet B., Caractérisation de l'emmental " grand-cru » français, I. Composition physicochimique, Lait 70 (1990) 1-14. 
[6] Biede S.L., Hammond E.G., Swiss cheese flavor: I. Chemical analysis, J. Dairy Sci. 62 (1979) 227-237.

[7] Bütikofer U., Fuchs D., Development of free amino acids in Appenzeller, Emmentaler. Gruyère, Raclette, Sbrinz and Tilsiter cheese, Lait 77 (1997) 91-100.

[8] Chamba J.F., Interaction entre lactobacilles thermophiles et bactéries propioniques, Influence sur les caractéristiques de l'emmental, in: Actes du Colloque Lactic 94, Caen, France, 7-9 septembre, 1994.

[9] Chamba J.F., Prost F., Les fromages à pâte pressée cuite, in: Bourgeois C.M., Larpent J.P. (Eds), Microbiologie alimentaire, Lavoisier, Paris, 1989, 232-246.

[10] Crow V.L., Metabolism of aspartate by Propionibacterium freudenreichii subsp. shermanii: Effect on lactate fermentation, Appl. Environ Microbiol. 52 (1986) 359-365.

[11] Crow V.L., Turner K.W., The effect of succinate production on other fermentation products in Swiss-type cheese, NZ J. Dairy Sci. Technol. 21(1986) 217-227.

[12] Crow V.L., Coolbear T., Gopal P.K., Martley F.G., McKay L.L., Riepe A., The role of autolysis of lactic acid bacteria in the ripening of cheese, Int. Dairy J. 5 (1995) 855-875.

[13] Demarigny Y., Beuvier E., Dasen A., Duboz G. Influence of raw milk microflora on the characteristics of Swiss-type cheeses, I. Evolution of microflora during ripening and characterization of facultatively heterofermentative lactobacilli, Lait 76 (1996) 371-387.

[14] Demeter K.J., Janoschek A., Günther E., Weiteres über die Bakteriologie der Emmentalerkäse-Bereitung und -Reifung, Milchwissenschaft 8 (1953) 420-426.

[15] Dicks L.M.T., Du Plessis E.M., Dellaglio F., Lauer E., Reclassment of Lactobacillus casei subsp. casei ATCC 393 and Lactobacillus rhamnosus ATCC 15820 as Lactobacillus zeae nom, rev. Designation of ATCC 334 as the neotype of Lactobacillus casei subsp. casei and rejection of the name Lactobacillus paracasei, Int. J .Syst. Bacteriol. 46 (1996) 337-340.

[16] Farkye N.Y., Fox P.F., Observation on plasmin activity in cheese, J. Dairy Res. 57 (1990) 413-418.

[17] FIL, Norme 33C Détermination de la teneur en phosphore total dans les fromages, Fédération Internationale de Laiterie, Bruxelles, 1987.

[18] FIL, Norme internationale 117A Yaourt. Dénombrement des micro-organismes caractéristiques, Fédération Internationale de Laiterie, Bruxelles, 1988

[19] FIL, Norme 135B Laits et produits laitiers. Caractéristiques de fidélité des méthodes analytiques, Fédération Internationale de Laiterie, Bruxelles, 1991.
[20] FIL, Norme 122B Laits et produits laitiers. Préparation des échantillons et des dilutions en vue de l'examen microbiologique, Fédération Internationale de Laiterie, Bruxelles, 1992.

[21] Fox P.F., Law J,, McSweeney P.L.H., Wallace J. Biochemistry of cheese ripening, in: Fox P,F. (ed), Cheese: chemistry, physics and microbiology, Elsevier, London, 1993, 389-438.

[22] Fox P.F., Wallace J.M., Morgan S., Lynch C.M., Niland E.J., Tobin J., Acceleration of cheese ripening, FEMS Microbiol. Rev. 70 (1996) 271-297.

[23] Gaucheron F., Le Graët Y., Piot M., Boyaval E., Determination of anions of milk by ion chromatography, Lait 76 (1996) 433-443.

[24] Gripon J.C., Desmazeaud M.J., Le Bars D., Bergère J.L., Étude du rôle des micro-organismes et des enzymes au cours de la maturation des fromages, Lait 55 (1975) 502-516.

[25] Gripon J.C., Mould-ripened cheeses, in: Fox P.F. (ed), Cheese: chemistry, physics and microbiology, Major cheese groups, vol 2, Elsevier, London, 1993, 111-136.

[26] Isolini D., Grand M., Glättli H., Selektivmedien zum Nachweis von obligat und fakultativ heterofermentativen Laktobazillen, Schweiz. Milchw. Forchung 19 (1990) 57-59.

[27] ITG, Synthèse actuelle des connaissances sur l'affinage, Étude ITG SS1984/07.08/C, Institut Technique du Gruyère, La Roche-sur-Foron, 1984.

[28] Jarret W.D., Aston J.W., Dulley J.R., A simple method for estimating free amino acids in Cheddar cheese, Aust J. Dairy Technol. 37 (1982) 55-58.

[29] Jimeno J., Lazaro M.J., Sollberger H., Antagonistic interactions between propionic acid bacteria and non-starter lactic acid bacteria, Lait 75 (1995) 401-413.

[30] Jordan K.N., Cogan T.M., Identification and growth of non-starter lactic acid bacteria in Irish cheddar cheese, Irish J. Agric. Food Res, 32 (1993) 47-55,

[31] Kiuru V.J.T., Tybeck E., Virtanen A.I., Free amino acids in Emmental cheese at different stages of ripening, Suom Kemistilehti 26B (1953) $55-56$.

[32] Langsrud T., Reinbold G.W., Flavor development and microbiology of Swiss cheese - A review, III. Ripening and flavor production, J. Milk Food Technol. 36 (1973) 593-609.

[33] Lavanchy P., Sieber R., Proteolyse in verschiedenen Hart- und Halbhartkäsen: 1. freie Aminosäuren, Schweiz. Milchw. Forschung 22 (1993) 59-64.

[34] Lindberg A.M., Christiansson A., Rukke E.O., Eklund T., Molin G., Bacterial flora of Norwegian and Swedish semi-hard cheese after ripening, with special reference to Lactobacillus, Neth Milk Dairy J. 50 (1996) 563-572. 
[35] Lortal S., Lemée R., Valence F., Autolysis of thermophilic lactobacilli and dairy propionibacteria: a review, Lait 77 (1997) 133-150.

[36] Lucey J.A., Gorry C., Fox P.F., Changes in the acid-base buffering curves during the ripening of emmental cheese. Milchwissenschaft 48 (1993) 183-186.

[37] Manca de Nadra M.C., Pesce de Ruiz Holgado A.A., Oliver G., Arginine dihydrolase activity in lactic acid bacteria, Milchwissenschaft 37 (1982) 669-670.

[38] Martley F.G., Crow V.L., Interactions between non-starter microorganisms during cheese manufacture and ripening, Int. Dairy J. 3 (1993) $461-483$.

[39] Mocquot G., Reviews of the progress of Dairy Science: Swiss-type cheese, J. Dairy Res. 46 (1979) 133-160.

[40] Peterson S.D., Marshall R.T., Non-starter lactobacilli in cheddar cheese: a review, J. Dairy Sci. 73 (1990) 1395-1410.

[41] Rohm H., Tschager E., Jaros D., Determination of proteolysis in Swiss cheese: Comparison of the Kjeldahl method and a spectrophotometric OPA assay, Lebensm. Wiss. Technol. 29 (1996) 191-194.

[42] Salvat-Brunaud D., Maubois J.L., Le Graët Y., Piot M., Maillard M.B., Corre C., Thierry A., Extraction et analyse de la phase aqueuse de l'emmental à 4 stades d'affinage, Lait 75 (1995) 239-249.
[43] SAS Institute Inc., SAS/STAT User's Guide, Release 6.03 Edition. Cary, NC: SAS Institute Inc, 1988.

[44] Sharpe M.E., Enumeration and studies of lactobacilli in food products, Dairy Sci. Abstr. 24 (1962) 165-171.

[45] SIGF, Statistiques pâtes pressées cuites 1996 , Syndicat Interprofessionnel du Gruyère Français, Paris, France, 1997.

[46] Sollberger H., Parameter zur Früherfassung des Nachgärungsrisikos beim Emmentaler-Käse, Schweiz. Milchw. Forschung 23 (1994) 20-24.

[47] Steffen C., Eberhard P., Bosset J.O., Rüegg M., Swiss-type varieties, in: P.F. Fox (ed.), Cheese: chemistry, physics and microbiology, Major cheese groups, vol 2, Elsevier, London, 1993, pp. 83-110.

[48] Thierry A., Madec M.N., Richoux R., Croissance des bactéries propioniques dans le fromage : comparaison de 2 milieux de dénombrement, Lait 74 (1994) 161-171.

[49] Thierry A., Madec M.N., Enumeration of propionibacteria in raw milk using a new selective medium, Lait 75 (1995) 315-323.

[50] Veaux M., Accolas J.P., Vassal L., Auclair J., Évolution des bactéries lactiques et des bactéries propioniques au cours de la fabrication et de l'affinage du Gruyère, in: Brèves comm XIX Congr. Int. Lait., 1974, pp. 458-459.

[51] Zoon P., Allersma D., Eye and crack formation in cheese by carbon dioxide from decarboxylation of glutamic acid, Neth. Milk Dairy J. 50 (1996) 309-318. 